\title{
F-JUMPING AND F-JACOBIAN IDEALS FOR HYPERSURFACES
}

\author{
LUIS NÚÑEZ-BETANCOURT AND FELIPE PÉREZ
}

\begin{abstract}
We introduce two families of ideals, $F$-jumping ideals and $F$-Jacobian ideals, in order to study the singularities of hypersurfaces in positive characteristic. Both families are defined using the $D$-modules $M_{\alpha}$ that were introduced by Blickle, Mustaţă and Smith. Using strong connections between $F$-jumping ideals and generalized test ideals, we give a characterization of $F$-jumping numbers for hypersurfaces via $D$-modules and $F$-modules. In addition, we use $F$-Jacobian ideals to study intrinsic properties of the singularities of hypersurfaces. In particular, we give conditions for $F$-regularity. Moreover, we prove several properties of $F$-Jacobian ideals that resemble those of Jacobian ideals of polynomials.
\end{abstract}

\section{Contents}

1. Introduction 1

2. Preliminaries 3

3. $\quad F$-jumping ideals and $F$-jumping numbers $\quad 7$

4. F-Jacobian ideals $\quad 14$

$\begin{array}{ll}\text { Acknowledgments } & 26\end{array}$

$\begin{array}{ll}\text { References } & 27\end{array}$

\section{INTRODUCTION}

Throughout this article $R$ denotes a commutative Noetherian ring of prime characteristic $p$. We say that $R$ is an $F$-finite ring if the Frobenius map is a finite morphism. This is a mild condition that holds, for example, when $R$ is essentially of finite type over a perfect field.

The study of singularities in positive characteristic has received a lot of attention over the last decade. Some of the main tools that are used in this area are the theory of $F^{e}$-modules of Lyubeznik [Lyu97], the theory of $D_{R}$-modules, and the more recent theory of Cartier Modules [BB11].

In this note we use these techniques to introduce two families of ideals, the $F$-jumping ideals and the F-Jacobian ideals, and show that both families measure singularities in positive characteristic. In order to describe these two families, we first need to recall the $F^{e}$-modules $M_{\alpha}$ introduced by Blickle, Mustaţă, and Smith [BMS09] (see Section 2.4 for details about $F^{e}$-modules). Given an $F$-finite, regular ring $R$ of positive characteristic $p$, an element $f \in R$ and a rational number $\alpha=\frac{r}{p^{e}-1}$, the $F^{e}$-module $M_{\alpha}$ is an $R$-module isomorphic to $R_{f}$, together with a twisted action of the Frobenius operator $F^{e}$ that depends on $\alpha$. Explicitly, if we write $M_{\alpha}=R_{f} \cdot e_{\alpha}$ then

$$
F^{e}\left(\frac{g}{f^{\ell}} \cdot e_{\alpha}\right)_{1}=\frac{g^{p^{e}}}{f^{p^{e} \ell+r}} \cdot e_{\alpha} .
$$

(C) 2015. This manuscript version is made available under the Elsevier user license

http://www.elsevier.com/open-access/userlicense/1.0/ 
Note that this action suggests that $e_{\alpha}$ behaves formally like $f^{-\alpha}$; hence, for most of our treatment, we think of $M_{\alpha}$ as the set of fractions $\frac{g}{f^{\ell}} \cdot f^{-\alpha}$. We recall that any $F^{e}$

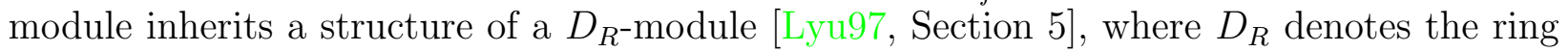
of differential operators of $R$ (see Section 2.3). Therefore every $F^{e}$-module $M_{\alpha}$ is a $D_{R^{-}}$ module as well.

$F$-jumping ideals: Let $R$ be an $F$-finite regular ring, $f$ an element of $R$ and $\alpha$ a positive real number. The test ideal $\tau\left(f^{\alpha}\right)$ was introduced by Hara and Yoshida as an analogue of the multiplier ideal in positive characteristic [HY03]. The $F$-jumping numbers for $f$ are defined as the positive real numbers $c$ such that $\tau\left(f^{c-\epsilon}\right) \neq \tau\left(f^{c}\right)$ for every $\epsilon>0$. These numbers have received a lot of attention since they encode important information about the singularities of $f$ (cf. [BFS13]). Blickle, Mustaţă and Smith showed that the $F$-jumping numbers are rational and form a discrete set [BMS09].

When $\alpha$ is a rational number such that $p$ does not divide its denominator, $\alpha$ can be written as $\frac{r}{p^{e}-1}$, with $r$ a positive integer. Then, we can consider the $F^{e}$-modules $M_{\alpha}$. Each $M_{\alpha}$ has a unique simple nonzero $F^{e}$-submodule $N_{\alpha}$, that is also the unique simple nonzero $D_{R}$-submodule (see Proposition 3.17 and Corollary 3.18). This leads to the definition of the $F$-jumping ideal $\mathfrak{J}_{F}\left(f^{\alpha}\right)$ as the ideal in $R$ such that

$$
\mathfrak{J}_{F}\left(f^{\alpha}\right) e_{\alpha}=R \cdot e_{\alpha} \cap N_{\alpha} .
$$

We determine when $\alpha$ is not an $F$-jumping number through the simplicity of $M_{\alpha}$, as well as through the properness of the ideal $\mathfrak{J}_{F}\left(f^{\alpha}\right)$, as follows:

Theorem 1.1 (see Theorem 3.22). Let $R$ be an F-finite regular domain and $f \in R$ be a nonzero element. Let $\alpha \in \mathbb{Q}$ be a rational number with denominator not divisible by $p$. Suppose that $\left(p^{e}-1\right) \alpha \in \mathbb{N}$. The following are equivalent:

(i) $\alpha$ is not an F-jumping number;

(ii) $N_{\alpha}=M_{\alpha}$;

(iii) $M_{\alpha}$ is a simple $D_{R}$-module;

(iv) $M_{\alpha}$ is a simple $F^{e}$-module (with the structure induced by the representation for $\alpha$ );

(v) $\mathfrak{J}_{F}\left(f^{\alpha}\right)=R$.

Moreover, we show that the test ideal $\tau\left(f^{\alpha}\right)$ is the minimal root for $N_{\alpha}$ and $\tau\left(f^{\alpha-\epsilon}\right)$ is the minimal root for $M_{\alpha}$ (see Propositions 3.19 and 3.20).

Remark 1.2. In characteristic zero Kashiwara proved that if $\alpha+m$ is not a root of the Bernstein-Sato polynomial of $f$ for $m \in \mathbb{Z}_{\geqslant 0}$, then $\mathcal{M}_{\alpha}:=D_{X} \cdot f^{\alpha}$ is irreducible ([Kas03, Corollary 6.25]). The implication (i) $\Rightarrow$ (iv) in the previous theorem can be viewed as an analogue, in positive characteristic, of this result.

F-Jacobian ideals: Suppose that $R=K\left[x_{1}, \ldots, x_{n}\right]$ is a polynomial ring over a perfect field and $f$ is an element in $R$. Recall that the Jacobian ideal of $f$ is defined by $\operatorname{Jac}(f)=$ $\left(f, \frac{\partial f}{\partial x_{1}}, \ldots, \frac{\partial f}{\partial_{x_{n}}}\right)$. This ideal plays a fundamental role in the study of singularities in both zero and positive characteristics. We recall that $\operatorname{Jac}(f)=R$ if and only if $R / f R$ is a regular ring. In addition, the Leibniz rule implies that $\operatorname{Jac}(f g) \subset f \operatorname{Jac}(g)+g \operatorname{Jac}(f)$ for $f, g \in R$. The equality in the previous containment holds only in specific cases [Fab13, Proposition 8], and it is used to study transversality of singular varieties and free divisors [Fab13, AF13]. To the best of our knowledge, these topics are widely open in positive characteristic. We now define an ideal that enjoys some similar features. 
In order to define the $F$-Jacobian ideals, we use Blickle's [Bli04] intersection homology $D_{R^{-}}$ module $\mathcal{L}(R / f R, R)$. This $D_{R}$-module is the sum of all the simple $D_{R}$-submodules of $R_{f} / R$. The $F$-Jacobian ideal of $f$, denoted by $J_{F}(f)$, is defined as the ideal in $R$ that contains $f$ and satisfies

$$
J_{F}(f) \frac{1}{f}=\mathcal{L}(R / f R, R) \cap R \frac{1}{f}
$$

in $R_{f} / R$. This ideal behaves similarly to the Jacobian ideal. For example, while the Jacobian ideal detects regularity, the $F$-Jacobian ideal detects $F$-regularity. More explicitly, if $R / f R$ is $F$-regular, then $J_{F}(f)=R$ (Corollary 4.29). In fact, if $R / f R$ is $F$-pure, then $R / f R$ is $F$-regular if and only if $J_{F}(f)=R$ (Corollary 4.31). We also show that, like the Jacobian ideal, the $F$-Jacobian ideal satisfies a Leibniz rule

$$
J_{F}(f g)=f J_{F}(g)+g J_{F}(f)
$$

for relatively prime elements $f, g \in R$ (Proposition 4.16). This is a key point, since it allows us to study interactions among different hypersurfaces.

The F-Jacobian ideals behave well with respect to $p^{e}$-th powers: $J_{F}\left(f^{p^{e}}\right)=J_{F}(f)^{\left[p^{e}\right]}$ (Proposition 4.32). This property is essential in several proofs and contrasts with how the Jacobian ideal changes with $p^{e}$-th powers:

$$
\operatorname{Jac}\left(f^{p^{e}}\right)=f^{p^{e}} R \neq\left(f^{p^{e}},\left(\frac{\partial f}{\partial x_{1}}\right)^{p^{e}}, \ldots,\left(\frac{\partial f}{\partial x_{n}}\right)^{p^{e}}\right)=\operatorname{Jac}(f)^{\left[p^{e}\right]} .
$$

The F-Jacobian ideal can be computed from the test ideal in certain cases and they are strongly related (see Proposition 4.26). However, they differ in general (see Example 4.43). Moreover, the $F$-Jacobian ideal can be defined for elements $f$ such that $R / f R$ is not reduced and satisfies properties that the test ideal does not (eg. Propositions 4.16 and 4.32). In Section 4.4, we show how the $F$-Jacobian arises in other contexts related with Cartier algebras, $F$-ideals, or $R\{F\}$-modules.

\section{PRELIMINARIES}

In this section we review the basic facts that we will need about tight closure, generalized test ideals, $D$-modules, and $F$-modules.

2.1. Tight closure. Tight closure was introduced by Hochster and Huneke [HH90, HH94a]. In this section we recall the basic definitions and mention some properties of this theory [Fed87, LS01]. Throughout this section, $R$ denotes a ring of positive characteristic $p$. For an ideal $I$, we set $I^{\left[p^{e}\right]}=\left(f^{p^{e}} \mid f \in I\right) R$.

If $R$ is reduced, $R^{1 / p}$ denotes the ring of $p$-roots of $R$. Note that $R$ is $F$-finite if and only if $R^{1 / p}$ is a finitely generated $R$-module. We say that $R$ is $F$-pure if for every $R$-module $M$ the morphism induced by the inclusion of $R$ in $R^{1 / p}, M \otimes_{R} R \rightarrow M \otimes_{R} R^{1 / p}$, is injective. If $R \rightarrow R^{1 / p}$ splits, we say that $R$ is $F$-split. These two properties, $F$-purity and $F$-splitting, are equivalent when $R$ is $F$-finite or complete. In addition, if $(R, \mathfrak{m}, K)$ is a regular local ring and $I \subset R$ is an ideal, then $R / I$ is $F$-pure if and only if $\left(I^{[p]}: I\right) \notin m^{[p]}$ (Fedder's Criterion, [Fed87, Theorem 1.12]). 
Let $I \subset R$ be an ideal. The tight closure $I^{*}$ of $I$ is the ideal of $R$ consisting of all elements $z \in R$ for which there exists some $c \in R$, not in any minimal prime of $R$, such that

$$
c z^{q} \in I^{[q]} \text { for all } q=p^{e} \gg 0 .
$$

We say that $R$ is weakly $F$-regular if $I=I^{*}$ for every ideal $I \subset R$. If every localization of $R$ is weakly $F$-regular, we say that $R$ is $F$-regular. In general, tight closure does not commute with localization [BM10] and it is unknown if the localization of a weakly $F$-regular ring is again a weakly $F$-regular ring. We say that $R$ is strongly $F$-regular if for each $c \in R$ that is not contained in any minimal prime of $R$, there exists $q=p^{e}$ such that the morphism of $R$-modules $R \rightarrow R^{1 / q}$ sending 1 to $c^{1 / q}$ splits. Strong $F$-regularity is preserved by localization.

We define the test ideal of $R$ by $\tau(R)=\bigcap_{I \subset R}\left(I: I^{*}\right)$. This ideal is also known in the literature as the finitistic test ideal or sometimes the classical test ideal. To avoid confusion, we recall that there exists another ideal, the big test ideal, which can be constructed without any mention of tight closure using Cartier operators. These two ideals are the same for Gorenstein rings [LS01]. Since we are only concerned with hypersurface rings, which are Gorenstein, we omit the adjectives finitistic and big for test ideals. Furthermore, in a Gorenstein ring $R$, strong and weak $F$-regularity are equivalent [LS01]. In addition, the test ideal can be computed as $\tau(R)=\bigcap_{I \text { parameter ideal }}\left(I: I^{*}\right)$ [HH90, Theorem 8.23]. If $R$ is also essentially of finite type over an excellent local ring, the test ideal commutes with localization [Smi94, Proposition 3.3] [LS01, Theorem 2.3]. As a consequence, $V(\tau(R))$ determines the prime ideals, $Q$, where $R_{Q}$ is not $F$-regular. Finally, we mention that $\tau(R)$ contains a nonzero-divisor for every excellent ring [HH94a, Theorem 6.1].

2.2. Generalized test ideals. Test ideals were generalized by Hara and Yoshida [HY03] to the context of pairs $\left(R, I^{c}\right)$, where $I$ is an ideal in $R$ and $c$ is a non-negative real number. In the case that $R$ is a regular $F$-finite ring, Blickle, Mustaţă, and Smith [BMS08] give an elementary description of these ideals. Below we use this description as our definition.

Given an ideal $I \subset R$, we denote by $I^{\left[1 / p^{e}\right]}$ the smallest ideal $J$ such that $I \subseteq J^{\left[p^{e}\right]}$ [BMS08, Definition 2.2]. The existence of such an ideal is not immediate, but it follows from the flatness of the Frobenius map for regular ring.

In the case when $I$ is a principal ideal we have the identity

$$
D^{(e)} f=\left((f)^{\left[1 / p^{e}\right]}\right)^{\left[p^{e}\right]}
$$

where $D^{(e)}=\operatorname{Hom}_{R^{p^{e}}}(R, R)$ (see [ÀMBL05, Proposition 3.1] and [BMS09, Lemma 2.2]).

Given a non-negative number $c$ and a nonzero ideal $I$ we define the generalized test ideal with exponent $c$ by

$$
\tau\left(I^{c}\right)=\bigcup_{e>0}\left(I^{\left\lceil c p^{e}\right\rceil}\right)^{\left[1 / p^{e}\right]}
$$

where $[c]$ stands for the smallest integer greater than or equal to $c$. This union is nested; hence, since the ring $R$ is Noetherian, there is an $e$ large enough such that $\tau\left(I^{c}\right)=\left(I^{\left\lceil c p^{e}\right\rceil}\right)^{\left[1 / p^{e}\right]}$. When $I=(f)$ is a principal ideal, we have that $\tau\left(f^{\frac{s}{p^{e}}}\right)=\left(f^{s}\right)^{\left[1 / p^{e}\right]}$ [BMS09, Lemma 2.1]. An important property of test ideals is given by the following proposition:

Proposition 2.1 (Skoda's Theorem [BMS08, Theorem 2.25]). If I is generated by s elements and $c \geqslant s$, then $\tau\left(I^{c}\right)=I \cdot \tau\left(I^{c-1}\right)$. 
For every nonzero ideal $I$ and every non-negative number $c$, there exists $\epsilon>0$ such that $\tau\left(I^{c}\right)=\tau\left(I^{c^{\prime}}\right)$ whenever $c<c^{\prime}<c+\epsilon$ [BMS08, Corollary 2.16]. A positive real number $c$ is an $F$-jumping number for $I$ if $\tau\left(I^{c}\right) \neq \tau\left(I^{c-\epsilon}\right)$ for all $\epsilon>0$. The $F$-jumping numbers of an ideal $I$ are rational and they form a discrete set [BMS08, Theorem 3.1]. Consequently, for every positive number $\alpha$ there is a positive rational number $\beta<\alpha$ such that $\tau\left(f^{\beta}\right)=\tau\left(f^{\gamma}\right)$ for any $\gamma \in(\beta, \alpha)$. We denote $\tau\left(f^{\beta}\right)$ by $\tau\left(f^{\alpha-\epsilon}\right)$.

2.3. $D$-modules. Let $R$ be a commutative ring and $A$ be subring of $R$. The elements of $\operatorname{Hom}_{A}(R, R)$ induced by multiplication by elements in $R$ are called $A$-linear differential operators of order zero. We say that $\theta \in \operatorname{Hom}_{A}(R, R)$ is an $A$-linear differential operator of order less than or equal to $k+1$, if for every $r \in R$ the element $\theta \cdot r-r \cdot \theta$ is an $A$-linear differential operator of order less than or equal to $k$. The subset of $\operatorname{Hom}_{A}(R, R)$ consisting of all $A$-linear differential operators forms a ring that we denote by $D(R, A)$. If $A=\mathbb{Z} / p \mathbb{Z}$, we write $D_{R}$ for $D(R, \mathbb{Z} / p \mathbb{Z})$, or just $D$ if $R$ is clear from the context.

If $A \subset B \subset R$ are rings, then $D(R, B) \subseteq D(R, A)$. If $M$ is a $D(R, A)$-module, then $M_{f}$ acquires a natural structure as a $D(R, A)$-module for all $f \in R$. Under this structure, the natural morphism $M \rightarrow M_{f}$ is a morphism of $D(R, A)$-modules. In particular, $R_{f} / R$ is a $D(R, A)$-module.

Assume $R$ is a reduced $F$-finite ring of positive characteristic $p$. For any multiplicative system $W \subset R$ and any simple $D_{R}$-module $M$, the $D_{R^{-m o d u l e}} W^{-1} M$ is either zero

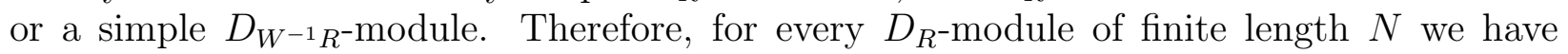
length $_{D_{W^{-1}} R} W^{-1} N \leqslant$ length $_{D_{R}} N$. Moreover, if $R$ is an $F$-finite domain, then $R$ is a strongly

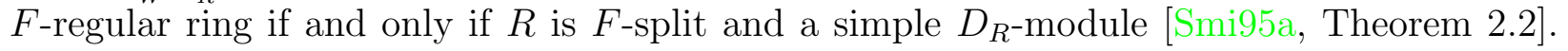
In addition, we have that $D_{R}=\bigcup_{e \in \mathbb{N}} \operatorname{Hom}_{R^{p}}(R, R)$ [Yek92]. We denote $\operatorname{Hom}_{R^{p^{e}}}(R, R)$ by $D_{R}^{(e)}$.

2.4. $F^{e}$-modules. The theory of $F$-modules was introduced by Lyubeznik [Lyu97]. In this section we recall the basic definitions and properties.

Every morphism of rings $\varphi: R \rightarrow S$ defines a functor from $R$-modules to $S$-modules $\varphi^{*}$, defined by $\varphi^{*} M=S \otimes_{R} M$. If $S=R$ and $\varphi$ is the Frobenius morphism, we denote by $F M$ the $R$-module $\varphi^{*} M$. This is the Frobenius functor introduced by Peskine and Szpiro [PS73]. If $R$ is regular, the flatness of Frobenius implies that $F$ is an exact functor. We denote the $e$-th iterated Frobenius functor by $F^{e}$.

Example 2.2. If $M$ is the cokernel of a matrix $\left(r_{i, j}\right)$, then $F^{e} M$ is the cokernel of $\left(r_{i, j}^{p^{e}}\right)$. In particular, if $I \subset R$ is an ideal, then $F^{e}(R / I)=R / I^{\left[p^{e}\right]}$.

An $F^{e}$-module is a pair $(\mathcal{M}, \nu)$ consisting of an $R$-module $\mathcal{M}$ and an isomorphism of $R$ modules $\nu: \mathcal{M} \rightarrow F^{e} \mathcal{M}$. The isomorphism $\nu$ is called the structural isomorphism. We note that an $F^{e}$-module has a natural structure as an $F^{\ell \cdot e}$-module for every $\ell \in \mathbb{N}$ given by the composition

$$
\mathcal{M} \stackrel{\nu}{\rightarrow} F^{e} \mathcal{M} \stackrel{F^{e} \nu}{\rightarrow} F^{2 e} \mathcal{M} \ldots \stackrel{F^{(\ell-1) e} \nu}{\rightarrow} F^{\ell \cdot e} \mathcal{M}
$$

One way to produce $F^{e}$-modules is by using generating morphisms. More explicitly, let $M$ be an $R$-module and $\beta: M \rightarrow F^{e} M$ be a morphism of $R$-modules. We consider

$$
\mathcal{M}=\lim _{\rightarrow}\left(M \stackrel{\beta}{\rightarrow} F^{e} M \stackrel{F^{e} \beta}{\rightarrow} F^{2 e} M \stackrel{F^{2 e} \beta}{\rightarrow} \ldots\right)
$$


and note that the Frobenius functor $F^{e}$ commutes with direct limits. Therefore,

$$
F^{e} \mathcal{M}=\lim _{\rightarrow}\left(F^{e} M \stackrel{F^{e} \beta}{\rightarrow} F^{2 e} M \stackrel{F^{2 e} \beta}{\rightarrow} F^{3 e} M \stackrel{F^{3 e} \beta}{\rightarrow} \ldots\right) \cong \mathcal{M}
$$

This gives $\mathcal{M}$ a structure of an $F^{e}$-module. In this case we say that $\mathcal{M}$ is generated by $\beta$, or that $\beta$ is a generating morphism for $\mathcal{M}$. If $\beta$ is an injective map, then $M$ injects into $\mathcal{M}$. In this case $\beta$ is called a root morphism for $\mathcal{M}$. If $\beta$ is understood from the context, we only say that $M$ is a root for $\mathcal{M}$. If there is a root $M$ which is finitely generated as an $R$-module, then $\mathcal{M}$ is called an $F^{e}$-finite $F^{e}$-module

Example 2.3. $R$ has a natural structure of an $F^{e}$-module for all $e$. Indeed, this follows from the fact that $F^{e} R=R$; hence, the structure morphism $\nu: R \rightarrow R$ is the identity map.

The next example plays an important role in Section 3.

Example 2.4 ([BMS09, Pag. 6653]). For every element $f \in R$ and $r, e \in \mathbb{N}$, we take $\alpha=\frac{r}{p^{e}-1}$ and define $M_{\alpha}$ as the $F^{e}$-finite $F^{e}$-module that is generated by

$$
R \stackrel{f^{r}}{\rightarrow} F^{e} R=R .
$$

The structure of $M_{\alpha}$ as $F^{e}$-module depends on the representation $\frac{r}{p^{e}-1}$. However, if we take another representation $\frac{r^{\prime}}{p^{e^{\prime}}-1}$, both structures will induce the same structure as $F^{e \cdot e^{\prime}}$-modules.

We say that $\phi: \mathcal{M} \rightarrow \mathcal{N}$ is a morphism of $F^{e}$-modules if the following diagram commutes:

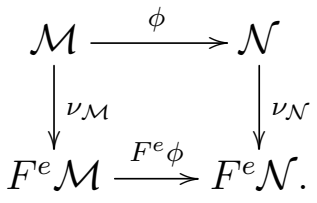

The $F^{e}$-modules form an Abelian category and the $F^{e}$-finite $F^{e}$-modules form a full Abelian subcategory. Moreover, if $\mathcal{M}$ is $F^{e}$-finite, then $\mathcal{M}_{f}$ is also $F^{e}$-finite.

Example 2.5. The localization map $R \rightarrow R_{f}$ is a morphism of $F$-modules for every $f \in R$. Moreover, the cokernel of the localization map $R \rightarrow R_{f}$ is an $F$-finite $F$-module for every $f \in R$. Indeed, $R_{f} / R=H_{f}^{1}(R)$ is generated by $R / f R \stackrel{f^{p-1}}{\rightarrow} F(R / f R)=R / f^{p} R$.

We recall that every $F^{e}$-submodule $M \subset R_{f} / R$ is a $D$-module [Lyu97, Examples 5.2]. We end this section presenting two important structural properties of $F$-modules.

Theorem 2.6 ([BB11, Theorem 5.13]). Every F-finite F-module over $R$ has finite length in the category of F-modules.

We refer to [Bli04, Lyu97] for the proof of the previous theorem in the local case.

Corollary 2.7. Every F-finite F-module over $R$ has finite length in the category of $D$ modules.

Proof. By Theorem 2.6, $R$ satisfies the hypotheses of [Lyu97, Theorem 5.6], which states that every simple $F$-module is a finite direct sum of simple $D$-modules. Then $M$ has finite length as a $D$-module. 


\section{3. $F$-JUMPING IDEALS AND $F$-JUMPING NUMBERS}

In this section we define $F$-jumping ideals and give some basic properties. In particular, we relate them with the generalized test ideals, $F$-jumping numbers, and the modules $M_{\alpha}$.

Notation 3.1. Throughout this section $R$ denotes an $F$-finite regular domain of characteristic $p>0$ and $\alpha$ denotes a rational number whose denominator is not divisible by $p$.

As the denominator of $\alpha$ is not divisible by $p$ we can write $\alpha=\frac{r}{p^{e}-1}$ for some $e>0$. An easy, but crucial consequence of this is that

$\left(p^{e(\ell-1)}+\ldots+p^{e}+1\right) r+\alpha=p^{e \ell} \alpha$ for every $\ell \in \mathbb{N}$.

Lemma 3.2. $\tau\left(f^{p \lambda}\right) \subset \tau\left(f^{\lambda}\right)^{[p]}$.

Proof. We have that $\frac{\left[p p^{j} \lambda\right]}{p^{j}} \leqslant \frac{p\left[p^{j} \lambda\right]}{p^{j}}$ and $\lim _{j \rightarrow \infty} \frac{p\left[p^{j} \lambda\right]}{p^{j}}=p \lambda$. Then,

$$
\tau\left(f^{p \lambda}\right)=\bigcup_{j \in \mathbb{N}}\left(f^{p\left[p^{j} \lambda\right]}\right)^{\left[1 / p^{j}\right]}=\bigcup_{j \in \mathbb{N}}\left(f^{\left[p^{j} \lambda\right]}\right)^{\left[1 / p^{j-1}\right]} \subset \bigcup_{j \in \mathbb{N}}\left(\left(f^{\left[p^{j} \lambda\right]}\right)^{\left[1 / p^{j}\right]}\right)^{[p]}=\tau\left(f^{\lambda}\right)^{[p]}
$$

by the properties of the ideals $I^{\left[1 / p^{j}\right]}[\mathrm{BMS} 08$, Lemma 2.4].

Recall from Example 2.4 that

$$
M_{\alpha}=\lim _{\rightarrow} R \stackrel{f^{r}}{\rightarrow} F^{e} R=R \stackrel{f^{p^{e}}}{\rightarrow} F^{2 e}(R)=R \stackrel{f^{p^{2 e}}}{\rightarrow} \ldots
$$

Hence, as an $R_{f}$-module, $M_{\alpha}$ is free of rank one. Let $e_{\alpha}$ denote the generator obtained as the image of $1 \in F^{0} R=R$ in the previous direct limit. We think of $e_{\alpha}$ as $1 / f^{\alpha}$.

$M_{\alpha}$ carries a natural structure as a $D$-module, which does not depend of the presentation of $\alpha$ [BMS09, Remark 2.4]. Given $P \in D_{R}^{s e}$, it is shown in loc. cit. that

$$
P \cdot\left(\frac{c}{f^{m}} \cdot e_{\alpha}\right)=\frac{P\left(c f^{m\left(p^{e s}-1\right)+r\left(1+p^{e}+\ldots+p^{e(s-1)}\right)}\right)}{f^{m p^{e s}+r\left(1+p^{e}+\ldots p^{e(s-1)}\right)}} \cdot e_{\alpha} .
$$

When $m=0$, the previous expression is equal to

$$
P \cdot\left(c \cdot e_{\alpha}\right)=\frac{P\left(c f^{r\left(1+p^{e}+\ldots p^{e(s-1)}\right)}\right)}{f^{r\left(1+p^{e}+\ldots+p^{e(s-1)}\right)}} \cdot e_{\alpha} .
$$

We also recall that $e_{\alpha}$ generates $M_{\alpha}$ as a $D$-module [BMS09, Theorem 2.11].

We now are ready to introduce the $F$-jumping ideals in terms of the $D$-module structure of $M_{\alpha}$.

Definition 3.3. Let $N_{\alpha}=D_{R} f^{\lceil\alpha\rceil} \cdot e_{\alpha}$, which is the smallest $D$-submodule of $M_{\alpha}$ containing $f^{[\alpha]} e_{\alpha}$. We define the $F$-jumping ideal associated to $f$ and $\alpha$ as the ideal $\mathfrak{J}_{F_{R}}\left(f^{\alpha}\right)$ of $R$ such that $\mathfrak{J}_{F_{R}}\left(f^{\alpha}\right) \cdot e_{\alpha}=N_{\alpha} \cap R \cdot e_{\alpha}$. Whenever the ring is clear from the context, we simply write $\mathfrak{J}_{F}\left(f^{\alpha}\right)$.

Lemma 3.4. Given any ideal $I \subseteq R$, the D-module generated by $I e_{\alpha}$ is

$$
D_{R} \cdot I e_{\alpha}=\bigcup_{s \geqslant 0}\left(D^{(e s)}\left(f^{r\left(1+\ldots+p^{e(s-1)}\right)} I\right) / f^{r\left(1+\ldots+p^{e(s-1)}\right)}\right) \cdot e_{\alpha}
$$


The intersection of this $D_{R}$-module with $R e_{\alpha}$ is equal to $J e_{\alpha}$, where

$$
J=\bigcup_{s \geqslant 0}\left(D_{R}^{(e s)}\left(f^{r\left(1+\ldots+p^{e(s-1)}\right)} I\right): f^{r\left(1+\ldots+p^{e(s-1)}\right)}\right) .
$$

In particular,

$$
\mathfrak{J}_{F_{R}}\left(f^{\alpha}\right)=\bigcup_{s \geqslant 0}\left(D^{(e s)}\left(f^{r\left(1+\ldots+p^{e(s-1)}\right)} f^{\lceil\alpha\rceil}\right): f^{r\left(1+\ldots+p^{e(s-1)}\right)}\right) .
$$

Proof. This follows immediately from the description of the action of the differential operators in $M_{\alpha}$.

For test ideals, if $\alpha<\beta$ then $\tau\left(f^{\alpha}\right) \supseteq \tau\left(f^{\beta}\right)$. The same statement is not true for the $F$-jumping ideals, but we can give a relation if $\beta=\alpha+l$ for $l \in \mathbb{N}$. Before stating this result, we need a lemma.

Lemma 3.5. The morphism $M_{\alpha} \rightarrow M_{\alpha+1}$ defined by sending $e_{\alpha} \mapsto f e_{\alpha+1}$ is an isomorphism of $F^{e}$-modules (as well as of D-modules). In particular, $N_{\alpha} \cong N_{\alpha+1}=D_{R} f^{\lceil\alpha+1\rceil} \cdot e_{\alpha+1}$.

Proof. Consider the following commutative diagram of $R$-modules,

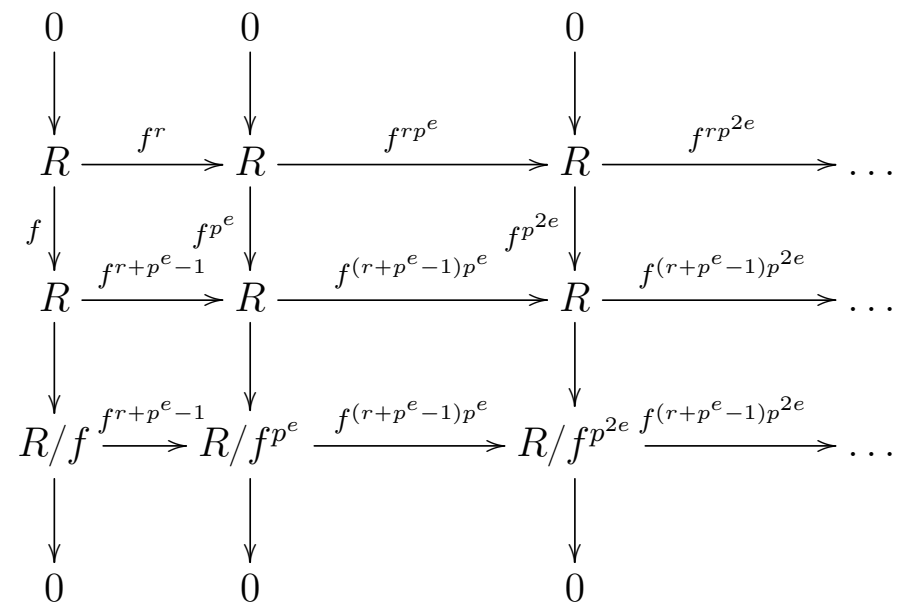

We note that $R / f \stackrel{f^{r+p^{e}-1}}{\rightarrow} R / f^{p^{e}}$ is the zero map because $r \geqslant 1$ (recall $\alpha>0$ ). By taking the limits, we obtain that the induced morphism $M_{\alpha} \rightarrow M_{\alpha+1}$ is an isomorphism of $F$-modules, and hence of $D$-modules.

Proposition 3.6. If $\ell \in \mathbb{N}$, then $f^{\ell} \mathfrak{J}_{F}\left(f^{\alpha}\right) \subset \mathfrak{J}_{F}\left(f^{\alpha+\ell}\right)$.

Proof. Let $\phi: M_{\alpha} \rightarrow M_{\alpha+\ell}$ be the morphism of $D$-modules given by $e_{\alpha} \mapsto f^{\ell} e_{\alpha+\ell}$. We know that $\phi$ is an isomorphism by Lemma 3.5. We obtain

$$
\begin{gathered}
f^{\ell} \mathfrak{J}_{F}\left(f^{\alpha}\right) e_{\alpha+\ell}=\mathfrak{J}_{F}\left(f^{\alpha}\right) f^{\ell} e_{\alpha+\ell}=\phi\left(\mathfrak{J}_{F}\left(f^{\alpha}\right) e_{\alpha}\right)=\phi\left(N_{\alpha} \cap R e_{\alpha}\right) \\
=\phi\left(N_{\alpha}\right) \cap \phi\left(R e_{\alpha}\right)=N_{\alpha+\ell} \cap R f^{\ell} e_{\alpha+\ell} \subset N_{\alpha+\ell} \cap R e_{\alpha+\ell}=\mathfrak{J}_{F}\left(f^{\alpha+\ell}\right) e_{\alpha+\ell} .
\end{gathered}
$$

We define inductively a sequence of ideals $\mathcal{I}_{R}^{j}\left(f^{\alpha}\right)$ associated to $f^{\alpha}$. These ideals will help us relate the test ideals to the $F$-jumping ideals. 
Definition 3.7. Given a representation of $\alpha=\frac{r}{p^{e}-1}$. We set $\mathcal{I}_{R}^{1}\left(f^{\alpha}\right)=\tau\left(f^{\alpha}\right)$. Given $\mathcal{I}_{R}^{j}\left(f^{\alpha}\right)$, we let

$$
\mathcal{I}^{j+1}\left(f^{\alpha}\right)=\left(\left(\mathcal{I}_{R}^{j}\left(f^{\alpha}\right)\right)^{\left[p^{e}\right]}: f^{r}\right) .
$$

If it is clear in which ring we are taking these ideals, we simply write $\mathcal{I}^{j}\left(f^{\alpha}\right)$.

We collect some basic properties of this sequence of ideals in the following proposition.

Proposition 3.8. If $\mathcal{I}_{R}^{j}\left(f^{\alpha}\right)$ is the sequence of ideals associated to $f \in R$ and $\alpha=\frac{r}{p^{e}-1}$, then for every positive integer $j$ :

(i) $\mathcal{I}_{R}^{j}\left(f^{\alpha}\right) \subset \mathcal{I}_{R}^{j+1}\left(f^{\alpha}\right)$, hence $\mathcal{I}_{R}^{n}\left(f^{\alpha}\right)=\mathcal{I}_{R}^{n+j}\left(f^{\alpha}\right)$ for some $n$ sufficiently large.

(ii) $f^{r} \mathcal{I}_{R}^{j+1}\left(f^{\alpha}\right) \subset \mathcal{I}_{R}^{j}\left(f^{\alpha}\right)^{\left[p^{e}\right]}$.

(iii) For all $j>1$ there is an equality $\mathcal{I}_{R}^{j}\left(f^{\alpha}\right)=\left(\tau\left(f^{\alpha}\right)^{\left[p^{j e}\right]}: f^{r\left(1+p^{e}+\ldots+p^{e(j-1)}\right)}\right)$.

Proof. We note that

$$
f^{r} \mathcal{I}^{1}\left(f^{\alpha}\right)=f^{r} \tau\left(f^{\alpha}\right)=\tau\left(f^{r+\alpha}\right)=\tau\left(f^{p^{e} \alpha}\right) \subset \tau\left(f^{\alpha}\right)^{\left[p^{e}\right]}=\mathcal{I}^{1}\left(f^{\alpha}\right)^{\left[p^{e}\right]} .
$$

The second equality follows from Skoda's Theorem (Proposition 2.1) and the last containment from Lemma 3.2. As a consequence, $\mathcal{I}^{1}\left(f^{\alpha}\right) \subset \mathcal{I}^{2}\left(f^{\alpha}\right)$ and $f^{r} \mathcal{I}^{2}\left(f^{\alpha}\right) \subset \mathcal{I}^{1}\left(f^{\alpha}\right)^{\left[p^{e}\right]}$. The assertions in (i) and (ii) follow by induction. For (iii) we use the properties of colon ideals and the flatness of Frobenius in a regular ring $R$.

Warning 3.9. As a consequence of the last part in the previous proposition, the flag of ideals associated to $f$ and $\alpha$ depends on the presentation of $\alpha=\frac{r}{p^{e}-1}$. However, the union of this flag does not depend on this presentation as the next proposition shows.

Proposition 3.10. $\mathfrak{J}_{F}\left(f^{\alpha}\right)=\bigcup_{j} \mathcal{I}^{j}\left(f^{\alpha}\right)=\mathcal{I}^{n}\left(f^{\alpha}\right)$ for $n \gg 0$.

Proof. By the definition of $\tau\left(f^{\alpha}\right)$ and properties of test ideals in Section 2.2, we have that, for a positive integer $n \in \mathbb{N}, \tau\left(f^{\alpha}\right)^{\left[p^{s e}\right]}=\left(\left(f^{\left[p^{e} \alpha\right]}\right)^{\left[1 / p^{s e}\right]}\right)^{\left[p^{s e}\right]}=D^{(e s)}\left(f^{\left[p^{s e} \alpha\right]}\right)$ for $s \geqslant n$. Then, by Lemma 3.4,

$$
\begin{aligned}
\mathfrak{J}_{F}\left(f^{\alpha}\right) e_{\alpha} & =N_{\alpha} \cap R e_{\alpha}=D_{R} f^{\lceil\alpha\rceil} \cdot e_{\alpha} \cap R e_{\alpha} \\
& =\bigcup_{s \geqslant 0}\left(D^{(e s)}\left(f^{r\left(1+\ldots+p^{e(s-1)}\right)} f^{\lceil\alpha\rceil}\right): f^{r\left(1+\ldots+p^{e(s-1)}\right)}\right) e_{\alpha} \\
& =\bigcup_{s \geqslant n}\left(D^{(e s)}\left(f^{r\left(1+\ldots+p^{e(s-1)}\right)} f^{\lceil\alpha\rceil}\right): f^{r\left(1+\ldots+p^{e(s-1)}\right)}\right) e_{\alpha} \\
& =\bigcup_{s \geqslant n}\left(D^{(e s)}\left(f^{\left\lceil r\left(1+\ldots+p^{e(s-1)}\right)+\alpha\right\rceil}\right): f^{r\left(1+\ldots+p^{e(s-1)}\right)}\right) e_{\alpha} \\
& =\bigcup_{s \geqslant n}\left(D^{(e s)}\left(f^{\left\lceil p^{e s} \alpha\right\rceil}\right): f^{r\left(1+\ldots+p^{e(s-1)}\right)}\right) e_{\alpha} \\
& =\bigcup_{s \geqslant n}\left(\tau\left(f^{\alpha}\right)^{\left[p^{s e}\right]}: f^{r\left(1+\ldots+p^{e(s-1)}\right)}\right) e_{\alpha}=\bigcup_{s \geqslant n} \mathcal{I}^{s}\left(f^{\alpha}\right) e_{\alpha} .
\end{aligned}
$$

The F-jumping ideals behave well with respect to localization and completion. 
Proposition 3.11. If $W \subset R$ is a multiplicative system in $R$, then $\mathfrak{J}_{F_{W^{-1}}}\left(f^{\alpha}\right)=W^{-1} \mathfrak{J}_{F_{R}}\left(f^{\alpha}\right)$. Proof. Choose $n$ such that $\mathfrak{J}_{F_{R}}\left(f^{\alpha}\right)=\mathcal{I}_{R}^{n}\left(f^{\alpha}\right)$. By Proposition 3.8(iii) we have

$$
\mathfrak{J}_{F_{R}}\left(f^{\alpha}\right)=\left(\tau\left(f^{\alpha}\right)^{\left[p^{j e}\right]}: f^{r\left(1+p^{e}+\ldots+p^{e(j-1)}\right)}\right)
$$

for all $j>n$. We have that test ideals commute with localization [BMS08, Proposition 2.13]. Since colon ideals and Frobenius powers of ideals commute with localization as well, the result follows from Proposition 3.10.

Proposition 3.12. If $R$ is a local ring and $\widehat{R}$ is the completion of $R$ with respect to the maximal ideal, then $\mathfrak{J}_{\hat{\widehat{R}}}\left(f^{\alpha}\right)=\mathfrak{J}_{F_{R}}\left(f^{\alpha}\right) \hat{R}$.

Proof. This proof is analogous to the proof of Proposition 3.11.

Notation 3.13. Every $F^{e}$-submodule of $M_{\alpha}$ is determined by an ideal $I \subset R$ such that we have an induced map $I \stackrel{f^{r}}{\rightarrow} I^{\left[p^{e}\right]}$, that is $f^{r} I \subset I^{\left[p^{e}\right]}$, [Lyu97, Corollary 2.6]. The $F^{e}$-submodule of $M_{\alpha}$ generated by $I$ is given by

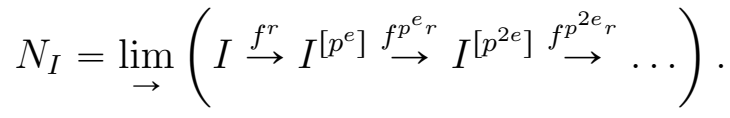

Lemma 3.14. If $I, J \subset R$ are ideals such that $f^{r} I \subset I^{\left[p^{e}\right]}, f^{r} J \subset J^{\left[p^{e}\right]}$, and $I \subset J$, then the $F^{e}$-submodule of $M_{\alpha}$ generated by $I$ is equal to the one generated by $J$ if and only if there exists $\ell \in \mathbb{N}$ such that $f^{r\left(1+\ldots+p^{e(\ell-1)}\right)} J \subset I^{\left[p^{p \ell}\right]}$.

Proof. Let $N_{I}$ and $N_{J}$ be the $F^{e}$-submodules of $M_{\alpha}$ generated by $I$ and $J$ respectively. We have that $J / I \stackrel{f^{r}}{\rightarrow} J^{\left[p^{e}\right]} / I^{\left[p^{e}\right]}$ generates the $F^{e}$-module $N_{J} / N_{I}$. Since $J / I$ is a finitely generated $R$-module, $N_{J}=N_{I}$ if and only if there exist $\ell$ such that

$$
J / I \stackrel{f^{r\left(1+\ldots+p^{e(\ell-1)}\right)}}{\rightarrow} J^{\left[p^{e \ell}\right]} / I^{\left[p^{e \ell}\right]}
$$

is the zero morphism. Therefore, $N_{I}=N_{J}$ if and only if there exists $\ell \in \mathbb{N}$ such that $f^{r\left(1+\ldots+p^{e(\ell-1)}\right)} J \subset I^{\left[p^{e}\right]}$.

Proposition 3.15. $N_{\alpha}$ is an $F^{e}$-submodule of $M_{\alpha}$. Moreover, the morphisms $\mathfrak{J}_{F}\left(f^{\alpha}\right) \stackrel{f^{r}}{\rightarrow}$ $\mathfrak{J}_{F}\left(f^{\alpha}\right)^{\left[p^{e}\right]}$ and $\tau\left(f^{\alpha}\right) \stackrel{f^{r}}{\rightarrow} \tau\left(f^{\alpha}\right)^{\left[p^{e}\right]}$ generate $N_{\alpha}$ as an $F^{e}$-module.

Proof. By the definition of $\tau\left(f^{\alpha}\right)$ and properties of test ideals in Section 2.2, there is an integer $\ell$ such that $\tau\left(f^{\alpha}\right)^{\left[p^{s e}\right]}=\left(\left(f^{\left[p^{s e} \alpha\right]}\right)^{\left[1 / p^{s e}\right]}\right)^{\left[p^{s e}\right]}=D^{(e s)}\left(f^{\left[p^{e s} \alpha\right]}\right)$ for all integers $s \geqslant \ell$. Then,

$$
\begin{aligned}
N_{\alpha}=D_{R} \cdot f^{[\alpha]} e_{\alpha} & =\bigcup_{s \geqslant 0} D^{(e s)} \cdot f^{[\alpha]} e_{\alpha}=\bigcup_{s \geqslant \ell} D^{(e s)} \cdot f^{[\alpha]} e_{\alpha} \\
& =\bigcup_{s \geqslant \ell}\left(D^{(e s)}\left(f^{r\left(1+\ldots+p^{e(s-1)}\right)} f^{[\alpha]}\right) / f^{r\left(1+\ldots+p^{e(s-1)}\right)}\right) \cdot e_{\alpha} \\
& =\bigcup_{s \geqslant \ell}\left(D^{(e s)}\left(f^{\left[p^{e s} \alpha\right]}\right) / f^{r\left(1+\ldots+p^{e(s-1)}\right)}\right) \cdot e_{\alpha} \\
& =\bigcup_{s \geqslant \ell}\left(\tau\left(f^{\alpha}\right)^{\left[p^{s e}\right]} / f^{r\left(1+\ldots+p^{e(s-1)}\right)}\right) \cdot e_{\alpha} .
\end{aligned}
$$


By Lemma 3.2, we have that $f^{r} \tau\left(f^{\alpha}\right)=\tau\left(f^{r+\alpha}\right)=\tau\left(f^{p^{e} \alpha}\right) \subset \tau\left(f^{\alpha}\right)^{\left[p^{e}\right]}$. Hence $\tau\left(f^{\alpha}\right) \stackrel{f^{r}}{\rightarrow}$ $\tau\left(f^{\alpha}\right)^{\left[p^{e}\right]}$ is a root for the $F^{e}$-submodule of $M_{\alpha}$

$$
\bigcup_{s}\left(\tau\left(f^{\alpha}\right)^{\left[p^{s e}\right]} / f^{r\left(1+\ldots+p^{e(s-1)}\right)}\right) \cdot e_{\alpha} .
$$

It follows that $N_{\alpha}$ is the $F^{e}$-submodule of $M_{\alpha}$ generated by the morphism $\tau\left(f^{\alpha}\right) \stackrel{f^{r}}{\rightarrow} \tau\left(f^{\alpha}\right)^{\left[p^{e}\right]}$.

For the second part, we note that $f^{r} \mathcal{I}^{s}\left(f^{\alpha}\right) \subset \mathcal{I}^{s}\left(f^{\alpha}\right)^{\left[p^{e}\right]}$ for every $s$ by Proposition 3.8 (i) and (ii). As a consequence, we deduce that $\mathcal{I}^{s}\left(f^{\alpha}\right)$ also generates an $F^{e}$-submodule of $M_{\alpha}$. By the same Proposition $f^{r} \mathcal{I}^{s+1}\left(f^{\alpha}\right) \subset \mathcal{I}^{s}\left(f^{\alpha}\right)^{\left[p^{e}\right]}$. Then the $F^{e}$-submodules generated by $\mathcal{I}^{s}\left(f^{\alpha}\right)$ are all the same for all $s$ by Lemma 3.14. As $\mathfrak{J}_{F}\left(f^{\alpha}\right)=\mathcal{I}^{s}\left(f^{\alpha}\right)$ for sufficiently large $s$, the result follows.

Lemma 3.16. Let $I \subset R$ be a nonzero ideal such that $I \subseteq\left(I^{\left[p^{e}\right]}: f^{r}\right)$. Then $f \in \sqrt{I}$.

Proof. The hypothesis is equivalent to $f^{r} I \subseteq I^{\left[p^{e}\right]}$. Let $P$ be a prime ideal of $R$. If $f \notin P$ then $I R_{P} \subseteq\left(I R_{P}\right)^{\left[p^{e}\right]}$. Therefore, by Nakayama's Lemma, we have $I R_{P}=R_{P}$. Hence if a prime ideal $P$ contains $I$, then it must contain $f$ as well. The result follows since $\sqrt{I}$ is the intersection of all prime ideals containing $I$.

Proposition 3.17. For any nonzero $F^{e}$-submodule $N$ of $M_{\alpha}, N_{\alpha} \subset N$. In particular, $N_{\alpha}$ is a simple $F^{e}$-module.

Proof. Since any two $R$-modules intersect in $R_{f}$, we deduce that there is a minimal simple $F^{e}$-submodule $N$. If $I$ is the ideal such that $R e_{\alpha} \bigcap N=I e_{\alpha}$, we have that $I=\left(I^{\left[p^{e}\right]}: f^{r}\right)$ [Lyu97, Corollary 2.6]. Moreover, $N=N_{I}$ (see Notation 3.13). Then $f^{n} \in I$ for some $n \in \mathbb{N}$ by Lemma 3.16.

Choose $\ell$ large enough such that $\tau\left(f^{\alpha}\right)^{\left[p^{s e}\right]}=\left(\left(f^{\left[p^{s e} \alpha\right]}\right)^{\left[1 / p^{s e}\right]}\right)^{\left[p^{s e}\right]}=D^{(e s)}\left(f^{\left[p^{s e} \alpha\right]}\right)$ and that $\tau\left(f^{\frac{n}{p s e}+\alpha}\right)=\tau\left(f^{\alpha}\right)$ for all $s \geqslant \ell$ (cf. Section 2.2). We have

$$
\begin{aligned}
& D_{R} \cdot f^{n} e_{\alpha}=\bigcup_{s \geqslant 0} D^{(e s)} \cdot f^{n} e_{\alpha}=\bigcup_{s \geqslant \ell} D^{(e s)} \cdot f^{n} e_{\alpha} \\
& =\bigcup_{s \geqslant \ell}\left(D^{(e s)}\left(f^{r\left(1+\ldots+p^{e(s-1)}\right)} f^{n}\right) / f^{r\left(1+\ldots+p^{e(s-1)}\right)}\right) \cdot e_{\alpha} \\
& =\bigcup_{s \geqslant \ell}\left(D^{(e s)}\left(f^{n+r\left(1+\ldots+p^{e(s-1)}\right)}\right) / f^{r\left(1+\ldots+p^{e(s-1)}\right)}\right) \cdot e_{\alpha} \\
& =\bigcup_{s \geqslant \ell}\left(\left(\left(f^{n+r\left(1+\ldots+p^{e(s-1)}\right)}\right)^{\left[1 / p^{s e}\right]}\right)^{\left[p^{e s}\right]} / f^{r\left(1+\ldots+p^{e(s-1)}\right)}\right) \cdot e_{\alpha}
\end{aligned}
$$

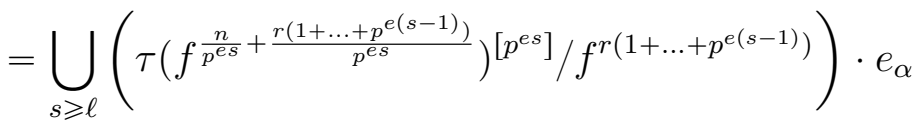

$$
\begin{aligned}
& \supset \bigcup_{s \geqslant 0}\left(\tau\left(f^{\frac{n}{p^{e s}}+\alpha}\right)^{\left[p^{e s}\right]} / f^{r\left(1+\ldots+p^{e(s-1)}\right)}\right) \cdot e_{\alpha} \\
& \supset \bigcup_{s \geqslant \ell}\left(\tau\left(f^{\alpha}\right)^{\left[p^{e s}\right]} / f^{r\left(1+\ldots+p^{e(s-1)}\right)}\right) \cdot e_{\alpha}=D_{R} \cdot f^{[\alpha]} e_{\alpha}=N_{\alpha} .
\end{aligned}
$$

Therefore, $N_{\alpha} \subset N$. 
Corollary 3.18. $N_{\alpha}$ is a simple D-module.

Proof. Since $N_{\alpha}$ is a simple $F^{e}$-module it is a direct sum of simple $D$-modules by Corollary 2.7. Using the fact that any two $R$-modules in $R_{f}$ intersect nontrivially, $N_{\alpha}$ must be a simple $D$-module.

Proposition 3.19. If $I \subset \tau\left(f^{\alpha}\right)$ is a nonzero ideal such that $I \subset\left(I^{\left[p^{e}\right]}: f^{r}\right)$, then $I=\tau\left(f^{\alpha}\right)$. In particular, $\tau\left(f^{\alpha}\right) e_{\alpha}$ is a minimal root for $N_{\alpha}$.

Proof. The flatness of Frobenius and the properties of colon ideals in the regular case imply that

$$
\left(I^{\left[p^{s e}\right]}: f^{r\left(1+p^{e}+\ldots+p^{e(s-1)}\right)}\right)=\left(\left(I^{\left[p^{(s-1) e}\right]}: f^{r\left(1+p^{e}+\ldots+p^{e(s-2)}\right)}\right)^{\left[p^{e}\right]}: f^{r}\right) \text {. }
$$

Hence, by an inductive argument, we deduce that $I \subset\left(I^{\left[p^{s e}\right]}: f^{r\left(1+p^{e}+\ldots+p^{e(s-1)}\right)}\right)$ for every $s \in \mathbb{N}$. There exists $n \in \mathbb{N}$ such that $f^{n} \in I$ by Lemma 3.16, and we can choose it such that $n>\alpha$. Hence $f^{n+\frac{p^{e s}-1}{p^{e}-1} r} \in I^{\left[p^{e s}\right]}$ which implies

$$
\left(f^{n+\frac{p^{e s}-1}{p^{e}-1} r}\right)^{\left[1 / p^{e s}\right]} \subset I .
$$

Note that $\left(p^{e}-1\right) n>r$. Thus,

$$
\frac{1}{p^{e s}}\left(n+\frac{p^{e s}-1}{p^{e}-1} r\right)=\frac{\left(p^{e}-1\right) n+\left(p^{e s}-1\right) r}{p^{e s}\left(p^{e}-1\right)}=\frac{\left(\left(p^{e}-1\right) n-r\right.}{p^{e s}\left(p^{e}-1\right)}+\frac{r}{p^{e}-1}>\frac{r}{p^{e}-1},
$$

and

$$
\lim _{s \rightarrow \infty} \frac{\left(p^{e}-1\right) n+\left(p^{e s}-1\right) r}{p^{e s}\left(p^{e}-1\right)}=\frac{r}{p^{e}-1}=\alpha .
$$

Then, for $\ell$ sufficiently large, $\tau\left(f^{\alpha}\right)=\left(f^{n+\frac{p^{e \ell}-1}{p^{e}-1} r}\right)^{\left[1 / p^{e \ell}\right]} \subset I \subset \tau\left(f^{\alpha}\right)$. Therefore $I=\tau\left(f^{\alpha}\right)$.

The claim about being a minimal root follows because $I \stackrel{f^{r}}{\rightarrow} I^{\left[p^{e}\right]}$ generates a nonzero $F^{e}$-submodule of $N_{\alpha}$ by Proposition 3.15, but $N_{\alpha}$ is simple by Proposition 3.17.

Using ideas analogous to the ones used in the previous proof, we recover a result previously obtained by Blickle [Bli08, Note before Proposition 3.5]

Proposition 3.20. Let $\beta=\frac{a}{b} \in \mathbb{Q}_{>0}$. If $\alpha>\beta$, then $f^{r} \tau\left(f^{\beta}\right) \subset \tau\left(f^{\beta}\right)^{\left[p^{e}\right]}$ and $\tau\left(f^{\beta}\right)$ generates $M_{\alpha}$ as $F^{e}$-module.

Proof. Since $\frac{r}{p^{e}-1}>\frac{a}{b}$, we have that $b r+a>p^{e} a$. Then

$$
f^{r} \tau\left(f^{\beta}\right)=\tau\left(f^{\beta+r}\right)=\tau\left(f^{\frac{a+b r}{b}}\right) \subset \tau\left(f^{\frac{p^{e} a}{b}}\right)=\tau\left(f^{p^{e} \beta}\right) \subset \tau\left(f^{\beta}\right)^{\left[p^{e}\right]},
$$

where the last containment follows from Lemma 3.2. Thus $\tau\left(f^{\beta}\right)$ generates an $F$-submodule of $M_{\alpha}$.

Clearly $\lim _{\ell \rightarrow \infty} \frac{r\left(1+\ldots+p^{e(\ell-1)}\right)}{p^{e \ell}}=\alpha$, so we can pick $\ell \in \mathbb{N}$ such that $r\left(1+\ldots+p^{e(\ell-1)}\right)>p^{e \ell} \beta$. With this choice of $\ell$, we have

$$
f^{r\left(1+\ldots+p^{e(\ell-1)}\right)} R \subset\left(\left(f^{r\left(1+\ldots+p^{e(\ell-1)}\right)}\right)^{\left[1 / p^{e l}\right]}\right)^{\left[p^{e l}\right]}=\tau\left(f^{\frac{r\left(1+\ldots+p^{e(\ell-1)}\right)}{p^{e \ell}}}\right)^{\left[p^{e l}\right]} .
$$

by properties of test ideals discussed in Section 2.2. 
Then $\tau\left(f^{\frac{r\left(1+\ldots+p^{e(\ell-1)}\right)}{p^{e \ell}}}\right)$ generates $M_{\alpha}$ as an $F^{e}$-module by Lemma 3.14. In view of $\tau\left(f^{\frac{r\left(p^{e(\ell-1)}+\ldots 1\right)}{p^{\ell \ell}}}\right) \subset \tau\left(f^{\beta}\right)$, we deduce that $\tau\left(f^{\beta}\right)$ also generates $M_{\alpha}$ as an $F^{e}$-module.

Remark 3.21. Blickle's result [Bli08, Proposition 3.5] shows more; the test ideal $\tau\left(f^{\alpha-\epsilon}\right)$ is a minimal root for the $F^{e}$-module $M_{\alpha}$.

Theorem 3.22. Let $R$ be an $F$-finite regular domain and $f \in R$ be a nonzero element. Let $\alpha \in \mathbb{Q}$ be a rational number with no positive power of $p$ in the denominator. Suppose that $\left(p^{e}-1\right) \alpha \in \mathbb{N}$. The following are equivalent:

(i) $\alpha$ is not an F-jumping number;

(ii) $N_{\alpha}=M_{\alpha}$;

(iii) $M_{\alpha}$ is a simple D-module;

(iv) $M_{\alpha}$ is a simple $F^{e}$-module (with the structure induced by the representation for $\alpha$ );

(v) $\mathfrak{J}_{F}\left(f^{\alpha}\right)=R$.

Proof. We have that $\alpha=\frac{r}{p^{e}-1}$ is not an $F$-jumping number if and only if $\tau\left(f^{\alpha}\right)=\tau\left(f^{\alpha-\epsilon}\right)$. This happens if and only if $N_{\alpha}=M_{\alpha}$ by Propositions 3.19 and 3.20 (see also Remark 3.21), which is equivalent to $\mathfrak{J}_{F}\left(f^{\alpha}\right)=R$. The rest follows from Proposition 3.17 and Corollary 3.18 .

Theorem 3.22 gives the following algorithm to decide whether a number of the form $\frac{r}{p^{e}-1}$ is an $F$-jumping number for $f \in R$ :

\section{Algorithm 3.23.}

Input: $\alpha=\frac{r}{p^{e}-1}$ and $f \in R$.

Output: $R$ if $\alpha$ is not $F$-jumping number for $f$ and a proper ideal otherwise.

Process: Compute $\tau\left(f^{\alpha}\right)$;

Take $J_{1}=\tau\left(f^{\alpha}\right)$;

Compute $J_{n+1}=\left(J_{n}^{\left[p^{e}\right]}: f^{r}\right)$ until there is an $\ell$ such that $J_{\ell}=J_{\ell+1}$;

Return: $J_{\ell}$.

Example 3.24. Let $R=\mathbb{F}_{13}[x, y]$ and $f=x^{2}+y^{3}$. Therefore:

(i) If $\alpha=\frac{11}{12}, \tau\left(f^{\alpha}\right)=(x, y) R, J_{1}=(x, y) R$, and $J_{2}=\left(\left(x^{13}, y^{13}\right): f^{11}\right)=R$ because $f^{11}$ is equal to $x^{22}-2 x^{20} y^{3}+3 x^{18} y^{6}-4 x^{16} y^{9}+5 x^{14} y^{12}-6 x^{12} y^{15}-6 x^{10} y^{18}+5 x^{8} y^{21}-$ $4 x^{6} y^{24}+3 x^{4} y^{27}-2 x^{2} y^{30}+y^{33}$. Then, $\frac{11}{12}$ is not an $F$-jumping number.

(ii) If $\alpha=\frac{10}{12}, \tau\left(f^{\alpha}\right)=(x, y) R, J_{1}=(x, y) R$, and $J_{2}=\left(\left(x^{13}, y^{13}\right): f^{10}\right)=(x, y)$ because $f^{10}$ is equal to $x^{20}-3 x^{18} y^{3}+6 x^{16} y^{6}+3 x^{14} y^{9}+2 x^{12} y^{12}+5 x^{10} y^{15}+2 x^{8} y^{18}+3 x^{6} y^{21}+$ $6 x^{4} y^{24}-3 x^{2} y^{27}+y^{30}$. Then, $\frac{10}{12}$ is an $F$-jumping number.

Proposition 3.25. $\sqrt{\mathfrak{J}_{F}\left(f^{\alpha}\right)}=\sqrt{\left(\tau\left(f^{\alpha}\right): \tau\left(f^{\alpha-\epsilon}\right)\right)}$.

Proof. We set a prime ideal $Q \subset R$. We have that $\mathfrak{J}_{F_{R_{Q}}}\left(f^{\alpha}\right)=\mathfrak{J}_{F_{R}}\left(f^{\alpha}\right) R_{Q}=R_{Q}$ if and only if $\alpha$ is not an $F$-jumping number for $f$ in $R_{Q}$. This is equivalent to $\left(\tau\left(f^{\alpha} R_{Q}\right): \tau\left(f^{\alpha-\epsilon} R_{Q}\right)\right)=$ $R_{Q}$. We have that $\operatorname{Supp}_{R} R / \mathfrak{J}_{F}\left(f^{\alpha}\right)=\operatorname{Supp}_{R} R /\left(\tau\left(f^{\alpha}\right): \tau\left(f^{\alpha-\epsilon}\right)\right)$. Then, both ideals give the set of prime ideals in which $\alpha$ is an $F$-jumping number for $f$. Hence, $\sqrt{\mathfrak{J}_{F}\left(f^{\alpha}\right)}=$ $\sqrt{\left(\tau\left(f^{\alpha}\right): \tau\left(f^{\alpha-\epsilon}\right)\right)}$. 
Remark 3.26. In general, $\mathfrak{J}_{F}\left(f^{\alpha}\right)$ is not equal to $\left(\tau\left(f^{\alpha}\right): \tau\left(f^{\beta}\right)\right)$. Let $R=\mathbb{F}_{7}[x, y], f=x^{3} y^{2}$ and $\alpha=\frac{4}{6}$. Then, $\beta=\frac{3}{6}$ is the biggest $F$-jumping number smaller than $\alpha$. We have that $\tau\left(f^{\beta}\right)=x y R$ and $\tau\left(f^{\alpha}\right)=x^{2} y R$, and so $\left(x^{2} y R: x y R\right)=x R$. However, $\mathfrak{J}_{F}\left(f^{\alpha}\right)=x^{2}$.

For test ideals, we have $\tau\left(f^{p \alpha}\right) \subset \tau\left(f^{\alpha}\right)^{[p]}$. We finish this section by showing that for $F$-jumping ideals a stronger result is true.

Lemma 3.27. $\mathcal{I}^{j}\left(f^{p^{e} \alpha}\right)=\mathcal{I}^{j-1}\left(f^{\alpha}\right)^{\left[p^{e}\right]}$ for $j \geqslant 2$.

Proof. We will prove that $\mathcal{I}^{j}\left(f^{p^{e} \alpha}\right)=\mathcal{I}^{j-1}\left(f^{\alpha}\right)^{\left[p^{e}\right]}$ for $j \geqslant 2$ by induction on $j$.

If $j=2$,

$$
\begin{gathered}
\mathcal{I}^{2}\left(f^{p^{e} \alpha}\right)=\mathcal{I}^{2}\left(f^{\alpha+r}\right)=\left(\tau\left(f^{\alpha+r}\right)^{\left[p^{e}\right]}: f^{p^{e} r}\right) \\
=\left(\tau\left(f^{\alpha+r}\right): f^{r}\right)^{\left[p^{e}\right]}=\left(f^{r} \tau\left(f^{\alpha}\right): f^{r}\right)^{\left[p^{e}\right]}=\tau\left(f^{\alpha}\right)^{\left[p^{e}\right]}=\mathcal{I}^{1}\left(f^{\alpha}\right)^{\left[p^{e}\right]} .
\end{gathered}
$$

Suppose that the assertion is true for $j$. Then,

$$
\begin{gathered}
\mathcal{I}^{j+1}\left(f^{p^{e} \alpha}\right)=\left(\mathcal{I}^{j}\left(f^{p^{e} \alpha}\right)^{\left[p^{e}\right]}: f^{p^{e} r}\right)=\left(\mathcal{I}^{j}\left(f^{p^{e} \alpha}\right): f^{r}\right)^{\left[p^{e}\right]} \\
=\left(\mathcal{I}^{j-1}\left(f^{\alpha}\right)^{\left[p^{e}\right]}: f^{r}\right)^{\left[p^{e}\right]}=\mathcal{I}^{j}\left(f^{\alpha}\right)^{\left[p^{e}\right]} .
\end{gathered}
$$

Proposition 3.28. $\mathfrak{J}_{F}\left(f^{p \alpha}\right)=\mathfrak{J}_{F}\left(f^{\alpha}\right)^{[p]}$.

Proof. We first note that $\mathfrak{J}_{F}\left(f^{p^{e} \alpha}\right)=\mathfrak{J}_{F}\left(f^{\alpha}\right)^{\left[p^{e}\right]}$ because

$$
\mathfrak{J}_{F}\left(f^{p^{e} \alpha}\right)=\bigcup_{j} \mathcal{I}^{j}\left(f^{p^{e} \alpha}\right)=\bigcup_{j} \mathcal{I}^{j}\left(f^{\alpha}\right)^{\left[p^{e}\right]}=\mathfrak{J}_{F}\left(f^{\alpha}\right)^{\left[p^{e}\right]}
$$

by Lemma 3.27. In addition, we have that $\mathfrak{J}_{F}\left(f^{\alpha}\right)^{[p]}=\left(\mathfrak{J}_{F}\left(f^{\alpha}\right)^{\left[p^{e+1}\right]}: f^{p r}\right)$. Then, $\mathfrak{J}_{F}\left(f^{\alpha}\right)^{[p]}$ defines the $F^{e}$-submodule of $M_{p \alpha}$ generated by the morphism $\mathfrak{J}_{F}\left(f^{\alpha}\right)^{[p]} \stackrel{f^{p r}}{\rightarrow} \mathfrak{J}_{F}\left(f^{\alpha}\right)^{\left[p^{e+1}\right]}$. Since $\mathfrak{J}_{F}\left(f^{p \alpha}\right)$ defines the unique simple $F$-submodule of $M_{p \alpha}$ by Propositions 3.15 and 3.17, $\mathfrak{J}_{F}\left(f^{p \alpha}\right) \subset \mathfrak{J}_{F}\left(f^{\alpha}\right)^{[p]}$. Combining these two observations we have that

$$
\mathfrak{J}_{F}\left(f^{p^{e} \alpha}\right) \subset \mathfrak{J}_{F}\left(f^{p^{e-1} \alpha}\right)^{[p]} \subset \ldots \subset \mathfrak{J}_{F}\left(f^{p \alpha}\right)^{\left[p^{e-1}\right]} \subset \mathfrak{J}_{F}\left(f^{\alpha}\right)^{\left[p^{e}\right]}=\mathfrak{J}_{F}\left(f^{p^{e} \alpha}\right) .
$$

Then all the containments are equalities. Hence, $\mathfrak{J}_{F}\left(f^{p \alpha}\right)=\mathfrak{J}_{F}\left(f^{\alpha}\right)^{[p]}$ because the Frobenius map is faithfully flat.

\section{F-JACOBIAN IDEALS}

The F-Jacobian ideal of an element $f$ is connected with the simple $F$-submodules of the first local cohomology of $R$ supported at $f R$. In this section, we extend the notion of the intersection homology for hypersurfaces introduced by Blickle [Bli04].

Notation 4.1. Throughout this section $R$ denotes an $F$-finite regular domain of characteristic $p>0$. For $f \in R$, let $\sigma: R / f R \rightarrow R_{f} / R$ be the morphism given by $\sigma([a])=a / f$ which is well defined because $R$ is a domain. Since the image of $\sigma$ is $R \frac{1}{f}$, we abuse notation and consider $R / f R \subset R_{f} / R$. 
4.1. Definition and first properties. In this subsection we define the F-Jacobian ideal and deduce some of its properties. We start with a few preparation lemmas.

Lemma 4.2. Let $f \in R$ be an element and $\pi: R \rightarrow R / f R$ be the quotient morphism. Let

$\mathcal{N}=\left\{N \subset R_{f} / R \mid N\right.$ is an F-submodule $\}$ and $\mathcal{I}=\left\{I \subset R \mid f \in I,\left(I^{[p]}: f^{p-1}\right)=I\right\}$.

Then, there is a bijective correspondence between $\mathcal{N}$ and $\mathcal{I}$, given by sending $N$ to $I_{N}=$ $\pi^{-1}(N \cap R / f R)$ with inverse defined by sending the ideal $I \in \mathcal{I}$ to the $F$-module $N_{I}$ generated by

$$
I / f R \stackrel{f^{p-1}}{\rightarrow} F(I / f R)=I^{[p]} / f^{p} R .
$$

Proof. Since $\phi: R / f R \stackrel{f^{p-1}}{\rightarrow} R / f^{p} R$ is a root for $R_{f} / R$, its $F$-submodules are in bijective correspondence with ideals $J \subset R / f R$ such that $\phi^{-1}(F(J))=J$ [Lyu97, Corollary 2.6].

If $J=I / f R$ for an ideal $I \subset R$, then $F(J)=I^{[p]} / f^{p} R$. Therefore,

$\phi^{-1}\left(I^{[p]} / f^{p}\right)=\left\{h \in R / f R \mid f^{p-1} h \in I^{[p]} / f^{p}\right\}=\left\{h \in R \mid f^{p-1} h \in I^{[p]}\right\} / f R=\left(I^{[p]}: f^{p-1}\right) / f R$ and the result follows.

Lemma 4.3. Suppose that $R$ is a UFD. Let $f \in R$ be an irreducible element. Then, $N \cap M \neq$ 0 for any nonzero $R$-submodules $M, N \subset R_{f} / R$.

Proof. Let $a / f^{\beta} \in M \backslash\{0\}$ and $b / f^{\gamma} \in N \backslash\{0\}$, where $\beta, \gamma \geqslant 1$. Since $R$ is a UFD and $f$ is irreducible, we may assume that $\operatorname{gcd}(a, f)=\operatorname{gcd}(b, f)=1$. Then, $\operatorname{gcd}(a b, f)=1$, and so $a b / f \neq 0$ in $R_{f} / R$. We have that $a b / f=b f^{\beta-1}\left(a / f^{\beta}\right)=a f^{\gamma-1}\left(b / f^{\gamma}\right) \neq 0$. Then, $a b / f \in N \cap M$.

Lemma 4.4. Suppose that $R$ is a local ring, and so, a UFD. If $f \in R$ is an irreducible element, then there is a unique simple $D$-submodule in $R_{f} / R$.

Proof. Since $R_{f} / R$ is an $D$-module of finite length, there exists a simple $D$-submodule $M \subset$ $R_{f} / R$. Let $N$ be a simple $D$-submodule of $R_{f} / R$. Since $M \cap N \neq 0$ by Lemma 4.3 and $M$ is a simple $D$-module, $M=M \cap N$. Likewise, $N=M \cap N$. Hence, $M=N$ is the only nonzero simple $D$-submodule of $R_{f} / R$.

Remark 4.5. Let $I \subset R$ an equidimensional ideal of codimension 1 . Let $Q_{1}, \ldots, Q_{\ell}$ be the minimal primes of $I$. By applying the Mayer-Vietoris sequence [BS13b, Chapter 3] several times, we obtain an inclusion

$$
H_{Q_{1}}^{1}(R) \oplus \ldots \oplus H_{Q_{\ell}}^{1}(R) \rightarrow H_{I}^{1}(R) .
$$

Let $\mathfrak{m} \subset R$ be any maximal ideal of $R$. Since $R_{\mathfrak{m}}$ is a UFD, there exist elements $g_{i}, f \in$ $R_{\mathfrak{m}}$ such that $Q_{i} R_{\mathfrak{m}}=g_{i} R_{\mathfrak{m}}, I R_{\mathfrak{m}}=f R_{\mathfrak{m}}$ and $f=g_{1} \cdots g_{\ell}$. Then, the map $H_{Q_{i}}^{1}\left(R_{\mathfrak{m}}\right) \rightarrow$ $H_{I}^{1}\left(R_{\mathfrak{m}}\right)$ is induced by the localization map $\left(R_{\mathfrak{m}}\right)_{g_{1}} \rightarrow\left(R_{\mathfrak{m}}\right)_{f}$. This has as a consequence that the previous inclusion is a morphism of $F$-modules and that $H_{Q_{i}}^{1}(R) \cong H_{Q_{i}}^{0} H_{I}^{1}(R)$ via the previous morphism because these two facts occur locally.

Propositions 4.6 and 4.9 are extensions of [Bli04, Theorem 4.1] for hypersurfaces. We point out that the proofs presented in this manuscript use neither étale invariance nor Kashiwara equivalence. 
Proposition 4.6. Let $Q$ be a prime ideal of height 1. Then, $H_{Q}^{1}(R)$ has a unique simple $D$-module, which is denoted by $\mathcal{L}(R / Q, R)$.

Proof. Recall that $H_{Q}^{1}(R)$ has finite length as an $F$-module by Theorem 2.6. Then, $H_{Q}^{1}(R)$ has finite length as a $D$-module by Corollary 2.7. We pick a simple $D$-submodule of $H_{Q}^{1}(R)$, and denote it by $N$. Let $M$ be also a simple $D$-submodule of $H_{Q}^{1}(R)$. We pick a maximal ideal $m \subset R$ such that $Q \subset m$. We note that $Q R_{\mathfrak{m}}$ is a principal ideal in $R_{\mathfrak{m}}$ because every regular local ring is a UFD. Therefore, we can find $g \in R_{\mathfrak{m}}$ such that $g R_{\mathfrak{m}}=Q R_{\mathfrak{m}}$. We note that $\operatorname{Ass}_{R} H_{Q}^{1}(R)=\{Q\}$. Therefore $\operatorname{Ass}_{R} N=\{Q\}$ because $N \neq 0$ and $\operatorname{Ass}_{R} N \subset \operatorname{Ass}_{R} H_{Q}^{1}(R)$. As a consequence, $N_{\mathfrak{m}} \neq 0$. Furthermore, length ${ }_{D_{R_{\mathfrak{m}}}} N_{\mathfrak{m}}=1$ because the length as a $D$ module cannot increase under localization. Then, $N_{\mathfrak{m}}$ is a simple $D_{R_{\mathfrak{m}}}$-submodule of $H_{g}^{1}\left(R_{\mathfrak{m}}\right)$. Similarly, $M_{\mathfrak{m}}$ is also a simple $D_{R_{\mathfrak{m}}}$-module of $H_{g}^{1}\left(R_{\mathfrak{m}}\right)$. We note that $g$ is an irreducible element because $(g)=Q R_{\mathfrak{m}}$ is a prime ideal. Hence, $M_{\mathfrak{m}} \cap N_{\mathfrak{m}} \neq 0$ and we have that $M_{\mathfrak{m}}=N_{\mathfrak{m}}$ by Lemma 4.4, which gives the result.

Blickle [Bli04] defined the intersection homology $D_{R}$-modules for a regular local ring of positive characteristic. We now present an extension of this notion to the case of any regular domain of positive characteristic.

Definition 4.7 (cf. [Bli04]). Let $I \subset R$ be an equi-dimensional ideal of codimension 1, and $Q_{1}, \ldots Q_{\ell} \in R$ be the minimal primes of $I$, We define the intersection homology $D$-module of $I$ by

$$
\mathcal{L}(R / I, R)=\sum_{i=1}^{\ell} \mathcal{L}\left(R / Q_{i}, R\right),
$$

where this sum is taken in $H_{I}^{1}(R)$ given that $H_{Q_{1}}^{1}(R) \cong H_{Q_{1}}^{0} H_{I}^{1}(R)$ by Remark 4.5.

Remark 4.8 (see [Bli04, Corollary 4.2]). Let $I \subset R$ be a equidimensional ideal of codimension 1. Let $N$ be a simple $D$-module of $H_{I}^{1}(R)$. Let $Q$ be an associated prime of $N$. Since $H_{Q}^{0}(N)$ is a nonzero $D$-submodule of $N$, we have that $N=H_{Q}^{0}(N)$. Then, $N$ is a simple $D$-submodule of $H_{Q}^{0} H_{I}^{1}(R)$; therefore, $N=\mathcal{L}(R / Q, R)$ by Remark 4.5 and Proposition 4.6. Hence, $\mathcal{L}(R / I, R)$ is the direct sum of all simple $D$-modules of $H_{I}^{1}(R)$ by the inclusion in Remark 4.5.

Proposition 4.9. Let $I \subset R$ be a equidimensional prime of codimension 1 . Then $\mathcal{L}(R / I, R)$ is an F-submodule of $H_{I}^{1}(R)$.

Proof. Since the sum of $F$-submodules is again an $F$-submodule, it suffices to prove the claim for prime ideals. Let $Q$ be a minimal prime ideal of height 1 and let $N$ denote the intersection homology $\mathcal{L}(R / Q, R)$.

Let $H_{Q}^{1}(R) \stackrel{\theta}{\rightarrow} F H_{Q}^{1}(R)$ be the structure morphism as an $F$-module. Let $\mathfrak{m} \subset R$ be a maximal ideal containing $Q$. Since $R_{\mathfrak{m}}$ is a UFD, there exists $g \in R_{\mathfrak{m}}$ such that $Q R_{\mathfrak{m}}=g R_{\mathfrak{m}}$. Since $N_{\mathfrak{m}}$ is the unique simple $D$-module of $H_{g}^{1}\left(R_{\mathfrak{m}}\right)$, this is equal to the intersection homology of $R / g R, \mathcal{L}\left(R_{\mathfrak{m}} / g R_{\mathfrak{m}}, R_{\mathfrak{m}}\right)$ [Bli04, Theorem 4.3]. Then, $N_{\mathfrak{m}}=\mathcal{L}\left(R_{\mathfrak{m}} / g R_{\mathfrak{m}}, R_{\mathfrak{m}}\right)$ is an $F$ submodule of $H_{g}^{1}(R)$.

We have that $\theta_{\mathfrak{m}}\left(N_{\mathfrak{m}}\right)=F N_{\mathfrak{m}}$ for every maximal ideal because $N$ is locally an $F$-submodule of $H_{Q}^{1}(R)$. Then, $\theta(N)=F N$ and $N$ is an $F$-submodule of $H_{Q}^{1}(R)$. 
Remark 4.10. Let $Q \subset R$ be a minimal prime of height 1 . Since $\mathcal{L}(R / Q, R)$ is a simple $D$ module and an $F$-finite $F$-module, we have that it must be a simple $F$-module by Corollary 2.7.

Remark 4.11. Suppose that $R$ is a UFD. As a consequence of Remark 4.8, we deduce that for $f=f_{1} \cdots f_{\ell}$ such that the $f_{1}, \ldots, f_{\ell}$ are relatively prime, then

$$
\mathcal{L}(R / f R, R)=\mathcal{L}\left(R / f_{1} R, R\right) \oplus \ldots \oplus \mathcal{L}\left(R / f_{\ell} R, R\right) \subseteq R_{f} / R .
$$

Indeed, this follows from the fact that the intersection of $R_{g}$ and $R_{h}$ in $R_{g h}$ is $R$ for all elements $g, h \in R$ such that $\operatorname{gcd}(g, h)=1$.

We are ready to introduce the main object studied in this section.

Definition 4.12. Let $f \in R$ be an element. $Q_{1}, \ldots Q_{\ell} \in R$ be the minimal primes of $R / f R$, and $\pi: R \rightarrow R / f R$ be the quotient morphism. We denote the pullback of $\mathcal{L}(R / f R, R) \cap R \frac{1}{f}$ to $R$ by $J_{F}(f)$, and we call it the $F$-Jacobian ideal of $f$. That is

$$
J_{F}(f)=\{a \in R \mid[a / f] \in \mathcal{L}(R / f R, R)\} .
$$

Proposition 4.13. Suppose that $R$ is a UFD. Let $g \in R$ be an irreducible element and $f=g^{n}$ for some integer $n \geqslant 1$. Then there exists a unique ideal $I \subset R$ such that:

(i) $f \in I$,

(ii) $I \neq f R$,

(iii) $\left(I^{[p]}: f^{p-1}\right)=I$, and

(iv) $I$ is contained in any other ideal satisfying (i),(ii) and (iii).

Proof. We note that $R_{f} / R=R_{g} / R$. Let $I$ be the ideal corresponding to the minimal simple $F$-submodule given in Lemma 4.4 under the bijection in Lemma 4.2. Then, it is clear from Lemma 4.2 that $I$ satisfies (i)-(iv).

Remark 4.14. Suppose that $R$ is a UFD. If $f \in R$ is an irreducible element, then:

(i) $\mathcal{L}(R / f R, R)=\mathcal{L}\left(R / f^{n} R, R\right)$ for every $n \in \mathbb{N}$ because $R_{f^{n}} / R=R_{f} / R$.

(ii) $J_{F}(f)$ is the minimal of the family of ideals $I$ containing properly $f R$ such that $\left(I: f^{p-1}\right)=I$ by Proposition 4.13.

(iii) $J_{F}(f)$ is not the usual Jacobian ideal of $f$. If $R=\mathbb{F}_{3}[x, y, z, w]$ and $f=x y+z w$, we have that the Jacobian of $f$ is $\mathfrak{m}=(x, y, z, w) S$. However, $\mathfrak{m} \neq\left(\mathfrak{m}^{[3]}: f^{2}\right)$.

(iv) $J_{F}(f)=R$ if and only if $R_{f} / R$ is a simple $F$-module by the proof of Proposition 4.13.

(v) $J_{F}(f)=R$ if and only if $R_{f} / R$ is a simple $D$-module by Proposition 4.9.

In order to reduce proofs to regular local rings, which are UFDs, we show that the $F$ Jacobian ideal commutes with localization.

Proposition 4.15. Let $f \in R$ and $W \subset R$ be a multiplicative system. Then, $J_{F_{W^{-1}}}(f)=$ $W^{-1} J_{F_{R}}(f)$.

Proof. Let $Q_{1}, \ldots, Q_{\ell}$ be the minimal primes of $f R$. We note that $W^{-1} \mathcal{L}\left(R / Q_{i} R, R\right)$ is zero if $Q_{i} \cap W \neq \varnothing$ or a simple $D$-submodule of $H_{f}^{1}\left(W^{-1} R\right)$ if $Q_{i} \cap W=\varnothing$ for $i=1, \ldots, \ell$. Hence, if $Q_{i} \cap W=\varnothing$, then $W^{-1} \mathcal{L}\left(R / Q_{i}, R\right)$ is the intersection homology of $W^{-1}\left(R / Q_{i}\right)$ by Proposition 4.6. As a consequence, $W^{-1} \mathcal{L}(R / f R, R)$ is the intersection homology of $W^{-1} R / f R$. Then,

$$
J_{F_{W^{-1}}}(f) / f W^{-1} R=W^{-1} R / f W_{17}^{-1} R \cap \mathcal{L}\left(W^{-1} R / f W^{-1} R, W^{-1} R\right)
$$




$$
=W^{-1}(R / f R \cap \mathcal{L}(R / f R, R))=W^{-1}\left(J_{F_{R}}(f) / f R\right),
$$

and the result follows because $f$ belongs to both ideals.

We now study how the $F$-Jacobian ideal interacts with different elements. In particular, we start by proving the Leibniz Rule for $F$-Jacobian ideals.

Proposition 4.16. Suppose that $R$ is a UFD. Let $f, g \in R$ be relatively prime elements. Then, $J_{F}(f g)=f J_{F}(g)+g J_{F}(f)$. Moreover, $f J_{F}(g) \cap g J_{F}(f)=f g R$.

Proof. We consider $R_{f} / R$ and $R_{g} / R$ as an $F$-submodules of $R_{f g} / R$, where the inclusion is given by the localization maps, $\iota_{f}: R_{f} \rightarrow R_{f g}$ and $\iota_{g}: R_{g} \rightarrow R_{f g}$.

Let $\pi: R \rightarrow R / f g R$ and $\rho: R \rightarrow R / f R$ be the quotient morphisms. The limit of the vertical morphism induced by the diagram

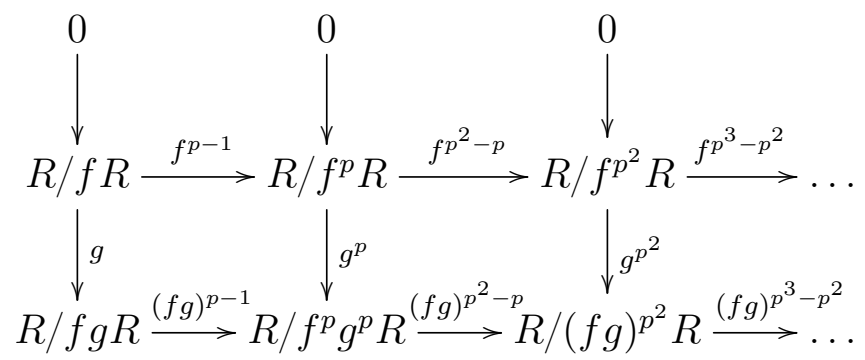

is induced by $\iota_{f}$. Moreover, under this correspondence

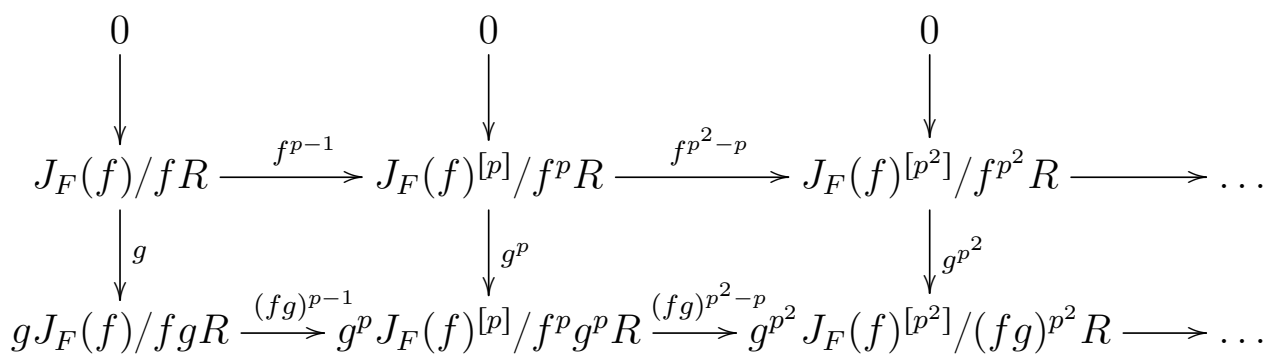

induces the isomorphism of $F$-modules, $\iota_{f}: \mathcal{L}(R / f R, R) \rightarrow \iota_{f}(\mathcal{L}(R / f R, R))$. We have that

$$
g J_{F}(f)=\pi^{-1}(\mathcal{L}(R / f R, R) \cap R / f g R) \subset \pi^{-1}(\mathcal{L}(R / f g R, R) \cap R / f g R)=J_{F}(f g) .
$$

In addition, $\left(g^{p} J_{F}(f)^{[p]}:(f g)^{p-1}\right)=g J_{F}(f)$, and it defines $\mathcal{L}(R / f R, R)$ as an $F$-submodule of $R_{f g} / R$. Likewise,

$$
f J_{F}(g) \subset J_{F}(f g),\left(f^{p} J_{F}(g)^{[p]}:(f g)^{p-1}\right)=f J_{F}(g),
$$

and it defines $\mathcal{L}(R / g R, R)$ as an $F$-submodule of $R_{f g} / R$. Then, $f J_{F}(g)+g J_{F}(f) \subset J_{F}(f g)$. Since $\mathcal{L}(R / f R, R) \cap \mathcal{L}(R / g R, R)=0$, we have that $f J_{F}(g) \cap g J_{F}(g)=f g R$.

We claim that $\left(f^{p} J_{F}(g)^{[p]}+g^{p} J_{F}(f)^{[p]}: f^{p-1} g^{p-1}\right)=f J_{F}(g)+g J_{F}(f)$.

To prove the first containment, take $h \in\left(f^{p} J_{F}(g)^{[p]}+g^{p} J_{F}(f)^{[p]}: f^{p-1} g^{p-1}\right)$. Then $f^{p-1} g^{p-1} h=f^{p} v+g^{p} w$ for some $v \in\left(J_{F}(g)\right)^{[p]}$ and $w \in J_{F}(g)^{[p]}$. Since $f$ and $g$ are relatively prime, $f^{p-1}$ divides $w$ and $g^{p-1}$ divides $v$. Thus, there exist $a, b \in R$ such that $v=g^{p-1} a$ and $w=g^{p-1} b$. Then, $a \in\left(J_{F}(g)^{[p]}: g^{p-1}\right)=J_{F}(g)$ and $b \in\left(J_{F}(f)^{[p]}: f^{p-1}\right)=J_{F}(f)$. Since $f^{p-1} g^{p-1} h=f^{p} v+g^{p} w=f^{p} g^{p-1} a+g^{p} f^{p-1} b$, we obtain that $h=f a+g b \in f J_{F}(g)+g J_{F}(f)$.

For the other containment, it is straightforward to check that

$$
f J_{F}(g)+g J_{F}(f) \subset\left(f^{p} J_{F}(g)^{[p]}+g^{p} J_{F}(f)^{[p]}: f^{p-1} g^{p-1}\right) .
$$


Since $N_{f J_{F}(g)+g J_{F}(f)}$, the $F$-module generated by $f J_{F}(g)+g J_{F}(f)$, contains $\mathcal{L}(R / f R, R)$ and $\mathcal{L}(R / g R, R)$, we have $\mathcal{L}(R / f R, R) \oplus \mathcal{L}(R / g R, R) \subset N_{f J_{F}(g)+g J_{F}(f)}$. Therefore, $J_{F}(f g) \subset$ $f J_{F}(g)+g J_{F}(f)$ and the result follows.

Proposition 4.17. Let $m, n \in \mathbb{N}$ be such that $m<n$. Then, $f^{n-m} J_{F}\left(f^{m}\right) \subset J_{F}\left(f^{n}\right) \subset$ $J_{F}\left(f^{m}\right)$.

Proof. From the definition of $F$-Jacobian ideal we have

$$
\begin{aligned}
f^{n-m} J_{F}\left(f^{m}\right) & =\left\{a \in R \mid f^{n-m} \cdot\left[a / f^{m}\right] \in \mathcal{L}(R / f R, R)\right\} \\
& \subset\left\{a \in R \mid\left[a / f^{n}\right] \in \mathcal{L}(R / f R, R)\right\} \\
& =J_{F}\left(f^{n}\right) .
\end{aligned}
$$

The second assertion can be proved in a similar way.

Remark 4.18. There are examples in which the containment in Proposition 4.17 is strict. Let $R=\mathbb{F}_{p}[x]$ and $f=x$. In this case, $R_{f} / R$ is a simple $F$-module. Then, $J_{F}\left(x^{m}\right)=R$ for every $m \in \mathbb{N}$ and $f^{n-m} J_{F}\left(f^{m}\right) \neq J_{F}\left(f^{n}\right)$ for every $n>m$.

Corollary 4.19. Let $f, g \in R$ be such that $f$ divides $g$. Then, $J_{F}(g) \subset J_{F}(f)$.

Proof. It suffices to prove the statement for local rings. We can assume that $R$ is a UFD. Then, the claim follows from Propositions 4.16 and 4.17.

4.2. Relations with test ideals and F-regularity. In this subsection we show properties of the F-Jacobian ideal in order to study singularities in positive characteristic.

Notation 4.20. If $f \in R$ is an element such that $R / f R$ is a reduced ring, we denote by $\tau_{f}$ the pullback of the test ideal of $R / f R, \tau(R / f R)$, to $R$.

Proposition 4.21. Suppose that $R$ is a UFD. Let $f \in R$ be an irreducible element. Then,

$$
J_{F}(f)=\bigcap_{\operatorname{gcd}(a, f)=1}\left(\bigcup_{e \in \mathbb{N}}\left(\left(\left(f^{p^{e}-1} a\right)^{\left[1 / p^{e}\right]}, f\right)^{\left[p^{e}\right]}: f^{p^{e}-1}\right)\right) .
$$

Proof. Since $R$ is a UFD and $f$ an irreducible element, we have that $\mathcal{L}(R / f R, R)$ is the only simple $D$-module of $R_{f} / R$. Then, $\mathcal{L}(R / f R, R)$ is the intersection of all nonzero cyclic $D$-modules generated by elements $a / f \in R_{f} / R$. Hence,

$$
J_{F}(f) / f=\bigcap_{\operatorname{gcd}(a, f)=1}((D \cdot a / f) \cap R \cdot 1 / f)=\bigcap_{\operatorname{gcd}(a, f)=1}\left(\bigcup_{e \in \mathbb{N}}\left(D^{(e)} \cdot a / f \cap R \cdot 1 / f\right)\right) .
$$

We have that $b \in J_{F}(f)$ if $b / f \in \mathcal{L}(R / f R, R)=\bigcap_{\operatorname{gcd}(a, f)=1} \bigcup_{e \in \mathbb{N}}\left(D^{(e)} \cdot a / f\right)$. Then there exists an $e \in \mathbb{N}$ such that $b / f \in D^{(e)} \cdot a / f$ for every $a \in R$ such that $\operatorname{gcd}(a, f)=1$. Thus, there exists $\phi \in D^{(e)}$ such that $\phi(a / f)=1 / f^{p^{e}} \phi\left(f^{p^{e}-1} a\right)=b / f$ in $R_{f} / R$. Then, there exists an element $r \in R$ such that $\phi(a / f)=1 / f^{p^{e}} \phi\left(f^{p^{e}-1} a\right)=b / f+r$ in $R_{f}$. Then, $f^{p^{e}-1} b=$ $\phi\left(f^{p^{e}-1} a\right)-f^{p^{e}} r$ and $b \in\left(I: f^{p^{e}-1}\right)$ for $I=D^{(e)} \cdot\left(f^{p^{e}-1} a\right)+f^{p^{e}} R$. Since $\left(\left(f^{p^{e}-1} a\right)^{\left[1 / p^{e}\right]}\right)^{\left[p^{e}\right]}=$ $D^{(e)}\left(f^{p^{e}-1} a\right)$, we have that $b \in \bigcap_{\operatorname{gcd}(a, f)=1}\left(\bigcup_{e \in \mathbb{N}}\left(\left(\left(f^{p^{e}-1} a\right)^{\left[1 / p^{e}\right]}, f\right)^{\left[p^{e}\right]}: f^{p^{e}-1}\right)\right)$. 
On the other hand, if

$$
b \in \bigcap_{\operatorname{gcd}(a, f)=1} \bigcup_{e \in \mathbb{N}}\left(\left(\left(f^{p^{e}-1} a\right)^{\left[1 / p^{e}\right]}, f\right)^{\left[p^{e}\right]}: f^{p^{e}-1}\right),
$$

then for every $a \in R$ such that $\operatorname{gcd}(a, f)=1$, there exist $e \in \mathbb{N}, \phi \in D^{(e)}$ and $r \in R$ such that $f^{p^{e}-1} b=\phi\left(f^{p^{e}-1} a\right)+f^{p^{e}} r$ because $\left(\left(f^{p^{e}-1} a\right)^{\left[1 / p^{e}\right]}\right)^{\left[p^{e}\right]}=D^{(e)}\left(f^{p^{e}-1} a\right)$. Therefore, after dividing by $f^{p^{e}}$, we have that $b / f \in D^{(e)} \cdot a / f$ in $R_{f} / R$. Hence, $b \in \bigcap_{\operatorname{gcd}(a, f)=1} \bigcup_{e \in \mathbb{N}}\left(D^{(e)} \cdot a / f\right)$, and $J_{F}(f)$.

We now present a theorem that relates the $F$-Jacobian ideal with $F$-regularity. We point out that this theorem is a consequence of a result of Blickle [Bli04, Corollary 4.10], as $R / f R$ is a Gorenstein ring. However, our proof is different from the one given there.

Theorem 4.22. Let $f \in R$ be such that $R / f R$ is an F-pure ring. If $J_{F}(f)=R$, then $R / f R$ is strongly F-regular.

Proof. We may assume that $(R, \mathfrak{m}, K)$ is local and $f \in \mathfrak{m}$, because $F$-purity and $F$-regularity are local properties for $R / f R$ and the $F$-Jacobian ideal commutes with localization by Proposition 4.15. We recall that every $F$-pure ring is reduced. Since $R / f R$ is reduced and $J_{F}(f)=R, f$ is irreducible by Proposition 4.16. Since $J_{F}(f)=R$, for every a such that $\operatorname{gcd}(a, f)=1$ there exists an $e \in \mathbb{N}$ such that $R=\left(\left(\left(f^{p^{e}-1} a\right)^{\left[1 / p^{e}\right]}, f\right)^{\left[p^{e}\right]}: f^{p^{e}-1}\right)$ by Lemma 4.21. Then, $f^{p^{e}-1} \in\left(\left(f^{p^{e}-1} a\right)^{\left[1 / p^{e}\right]}, f\right)^{\left[p^{e}\right]}$. Since $f^{p^{e}-1} \notin \mathfrak{m}^{\left[p^{e}\right]}$ for every $e \in \mathbb{N}$ by Fedder's criterion, $R=\left(f^{p^{e}-1} a\right)^{\left[1 / p^{e}\right]}$; otherwise, $\left(\left(f^{p^{e}-1} a\right)^{\left[1 / p^{e}\right]}, f\right) \subset \mathfrak{m}$. Then, there exists a morphism $\phi \in \operatorname{Hom}_{R^{p^{e}}}\left(R, R^{p^{e}}\right)$ such that $\phi\left(f^{p^{e}-1} a\right)=1$. Let $\varphi: R / f R \rightarrow R / f R$ be the morphism defined by $\varphi([x])=\left[\phi\left(f^{p^{e}-1} x\right)\right]$. This corresponds to an $R$-linear map $\varphi^{\prime}: R^{1 / p^{e}} \rightarrow R$ that sends $[a]^{1 / p^{e}} \rightarrow[1]$. Hence, $R / f R$ is strongly $F$-regular.

Remark 4.23. $J_{F_{R}}(f)=R$ does not imply that $R / f R$ is $F$-pure. Let $R=\mathbb{F}_{2}[x]$ and $f=x^{2}$. Then, $J_{F}(f)=R$ and $R / f R$ is not even reduced.

Remark 4.24. Blickle showed that the intersection homology of $f$ has as minimal root the test ideal of $R / f R$ [Bli04, Proposition 4.5]. As a consequence, we have that $f^{p-1} \tau_{f} \subset \tau_{f}^{[p]}$.

We now focus in proving relations between $J_{F}(f)$ and $\tau_{f}$.

Lemma 4.25. Suppose that $R / f R$ is reduced. Let $I^{j}(f)=\left(\tau_{f}^{\left[p^{j-1}\right]}: f^{p^{j-1}-1}\right)$. Then $I^{j}(f) \subset$ $I^{j+1}(f)$ and $I^{j+1}(f)=\left(I^{j}(f)^{[p]}: f^{p-1}\right)$. Moreover, $I^{j}(f) / f R \stackrel{f^{p-1}}{\rightarrow} I^{j}(f)^{[p]} / f^{p} R$ generates $\mathcal{L}(R / f R, R)$ as an F-module.

Proof. We note that $I^{1}(f)=\tau_{f}$ and it is a root for $\mathcal{L}(R / f R, R)$ by Remark 4.24. In addition, $f^{p-1} I^{1}(f)=f^{p-1} \tau_{f} \subset \tau_{f}^{\left[p^{e}\right]}=I^{1}(f)^{\left[p^{e}\right]}$. Thus, $I^{1}(f) \subset I^{2}(f)$ and $f^{p-1} I^{2}(f) \subset I_{1}(f)^{[p]}$. Moreover, $I^{2}(f) / f R$ is also a root for $\mathcal{L}(R / f R, R)$ because $I^{2}(f) / I^{1}(f)$ is the kernel of the $\operatorname{map} R / I^{1}(f) \stackrel{f^{p-1}}{\rightarrow} R / I^{1}(f)^{[p]}$. Inductively, we obtain that $I^{j}(f) \subset I^{j+1}(f), f^{r} I^{j+1}(f) \subset I^{j}(f)$ and that $I^{j}(f) / f R$ is a root for $\mathcal{L}(R / f R, R)$ for every $j \in \mathbb{N}$ and the result follows. 
The following proposition allows us to compute the $F$-Jacobian ideal from the classical test ideal.

Proposition 4.26. Suppose that $R / f R$ is reduced. Then, $J_{F}(f)=\bigcup_{j} I^{j}(f)$ and

$$
J_{F}(f) / f R \stackrel{f^{p-1}}{\rightarrow} J_{F}(f)^{[p]} / f^{p} R
$$

generates $\mathcal{L}(R / f R, R)$ as an $F$-module.

Proof. We have that the flag of ideals

$$
I^{1}(f) \subset I^{2}(f) \subset I^{3}(f) \subset \ldots
$$

eventually stops. Let $k$ be such that $I^{k}(f)=I^{j}(f)$ for $j \geqslant k$. Then, $I^{k}(f)=I^{k+1}(f)=$ $\left(I^{k}(f)^{[p]}: f^{p-1}\right)$ by Lemma 4.25. Thus, $I^{k}(f)$ is an ideal satisfying the conditions in Proposition 4.13, and so, $J_{f}(f) \subset I^{k}(f)$. Since $I^{k}(f) / f R \stackrel{f^{p-1}}{\rightarrow} I^{k}(f)^{[p]} / f^{p} R$ generates $\mathcal{L}(R / f R, R)$, we have that $I^{k}(f) / f R \subset \mathcal{L}(R / f R, R) \cap R / f R=J_{F}(f) / f R$. Hence,

$$
J_{F}(f)=I^{k}(f)=\bigcup_{j} I^{j}(f) .
$$

Remark 4.27. In general, we do not have $\tau_{f}=J_{F}(f)$. Let $R=K[x]$, where $K$ is any perfect field of characteristic $p>0$. Let $f=x^{2}$. Then, $\tau_{f}=x R \neq R=J_{F}(f)$. Example 4.43 shows another situation where $\tau_{f} \neq J_{F}(f)$.

Corollary 4.28. Suppose that $R$ is a $\mathbb{Z}^{h}$-graded UFD. Let $f \in R$ be a homogeneous element. Then, $J_{F}(f)$ is a homogeneous ideal.

Proof. It suffices to prove that $\mathcal{L}(R / f R, R)$ is a $\mathbb{Z}^{h}$-graded submodule of $R_{f} / R$. We can assume that $R / f R$ is reduced. We have that $\tau(R / f R)$ is a homogeneous ideal [HH94b, Theorem 4.2]. This means that $I_{R}^{j}(f)$ is a homogeneous ideal for every $j$. Therefore, $J_{F}(f)$ is homogeneous and that $\mathcal{L}(R / f R, R)$ is a $\mathbb{Z}^{n}$-graded submodule of $R_{f} / R$.

Corollary 4.29. Let $f \in R$ be such that $R / f R$ is reduced. Then,

$$
V\left(J_{F}(f)\right) \subset\left\{Q \in \operatorname{Spec}(R) \mid R_{Q} / f R_{Q} \text { is not F-regular }\right\} .
$$

Moreover, if $R / f R$ is an $F$-pure ring, then

$$
V\left(J_{F}(f)\right)=\left\{Q \in \operatorname{Spec}(R) \mid R_{Q} / f R_{Q} \text { is not F-regular }\right\} .
$$

Proof. For every prime ideal $Q \in V\left(J_{F}(f)\right), J_{F}(f) R_{Q}=J_{F_{R_{Q}}}(f) \neq R_{Q}$. We have that $\tau\left(R_{Q} / f R_{Q}\right) \subset J_{F_{R_{Q}}}(f) \subset Q R_{Q}$. Then, $R_{Q}$ is not $F$-regular.

Now, we suppose that $R / f R$ is $F$-pure. For every prime ideal $Q \subset R$ such that $R_{Q} / f R_{Q}$ is not $F$-regular. Then, $J_{F_{R_{P}}}(f) \neq R_{P}$ by Theorem 4.22. Then, $Q \in V\left(J_{F}(f)\right)$.

Lemma 4.30. Let $f \in R$ be an element. If $R / f R$ is $F$-pure, then $R / J_{F_{R}}(f)$ is $F$-pure.

Proof. We may assume $R$ is local with a maximal ideal $\mathfrak{m}$. Since $R / f R$ is $F$-pure, we have that $f^{p-1} \notin \mathfrak{m}^{[p]}$ by Fedder's Criterion. We have that $f^{p-1} \in\left(J_{F}(f)^{[p]}: J_{F}(f)\right)$, and so $\left(J_{F}(f)^{[p]}: J_{F}(f)\right) \notin \mathfrak{m}^{[p]}$. Therefore, $R / J_{F}(f)$ is $F$-pure.

Corollary 4.31. Let $f \in R$. If $R / f R$ is an F-pure ring, then $J_{F}(f)=\tau_{f}$. 
Proof. We have that $\sqrt{J_{F}(f)}=\sqrt{\tau_{f}}$ by Corollary 4.29 because

$$
V\left(\tau_{f}\right)=\left\{Q \in \operatorname{Spec}(R) \mid R_{Q} / f R_{Q} \text { is not } F \text {-regular }\right\}
$$

in this case. Since $R / J_{F}(f)$ is $F$-pure by Lemma $4.30, J_{F}(f)$ is a radical ideal. In addition, $\tau(R / f R)$ is a radical ideal [FW89, Proposition 2.5]. Therefore, $\tau_{f}$ is also a radical ideal. Hence, $J_{F}(f)=\tau_{f}$.

4.3. Behavior under extensions. In this subsection we study the behavior of $F$-Jacobian ideals under completion and flat extensions.

Proposition 4.32. Let $f \in R$. Then, $J_{F_{R^{1 / p}}}(f)=J_{F_{R}}(f) R^{1 / p}$. Equivalently, $J_{F_{R}}\left(f^{p}\right)=$ $J_{F_{R}}(f)^{[p]}$.

Proof. Since taking $p$-roots commutes with localization, it suffices to prove the statement for regular local rings. Then, we can assume that $R$ is a UFD. By Proposition 4.16, we may assume that $f=g^{n}$ where $g$ is irreducible. Let $h$ denote the length of $R_{f} / R$ in the category of $F$-modules. Let $G: R^{1 / p} \rightarrow R$ be the isomorphism defined by $r \rightarrow r^{p}$. Under the isomorphism $G, R_{f}^{1 / p} / R^{1 / p}$ corresponds to $R_{f^{p}} / R$. Then, the length of $R_{f}^{1 / p} / R^{1 / f}$ in the category of $F_{R^{1 / p}}$-modules is $h$. Let $0=M_{0} \subset \ldots \subset M_{h}=R_{f} / R$ be a chain of $F_{R^{\text {-submodules }}}$ of $R_{f} / R$ such that $M_{i+1} / M_{i}$ is a simple $F_{R}$-module. Let $f R=J_{0} \subset \ldots \subset J_{h}=R$ be the corresponding chain of ideals under the bijection given in Lemma 4.2. Since $f=g^{n}$ and $g$ is irreducible, $M_{1}=\mathcal{L}(R / f R, R)$ and $J_{1}=J_{F_{R}}(f)$. We note that $\left(J_{i}^{p} R^{1 / q}: f^{p-1} R^{1 / p}\right)=J_{i} R^{1 / p}$ and $J_{i} R^{1 / p} \neq J_{i+1} R^{1 / p}$ because $R^{1 / p}$ is a faithfully flat $R$-algebra.

Then, we have a strictly ascending chain of ideals

$$
f R^{1 / p}=J_{0} R^{1 / p} \subset \ldots \subset J_{h} R^{1 / p}=R^{1 / p}
$$

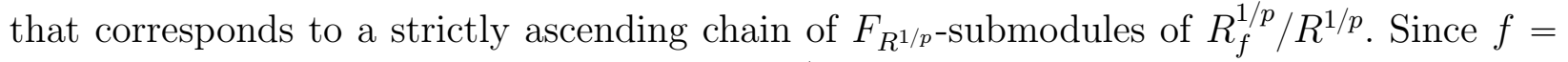
$\left(g^{1 / p}\right)^{p}, g^{1 / p}$ is irreducible and the length of $R_{f}^{1 / p} / R^{1 / p}$ is $h$, we have that

$$
J_{F_{R}}(f) R^{1 / p}=J_{1} R^{1 / p}=J_{F_{R^{1 / p}}}(f) .
$$

Therefore,

$$
J_{F_{R}}(f)^{[p]}=G\left(J_{F_{R}}(f) R^{1 / p}\right)=G\left(J_{F_{R^{1 / p}}}(f)\right)=J_{F_{R}}\left(f^{p}\right) .
$$

Proposition 4.33. Let $R \rightarrow S$ be a flat morphism of regular $F$-finite domains. Then $J_{F_{S}}(f) \subset J_{F_{R}}(f) S$.

Proof. Since flatness is a local property, it suffices to prove our claim for local rings. We may assume that $f=g^{\beta}$ where $g$ is an irreducible element in $R$ by Proposition 4.16. Since $S$ is flat, $\left(J_{F_{R}}(f)^{[p]} S: f^{p-1}\right)=J_{F_{R}}(f) S$. Let $M$ denote the $F_{S^{-}}$submodule of $S_{f} / S$ given by $J_{F_{R}}(f) S$ under the correspondence in Lemma 4.2. If $f$ is a unit in $S$, then $J_{F}(f) S=S$ and the result is immediate. We may assume that $f$ is not a unit in $S$. Since $J_{F}(f) \neq f R$, we can pick $a \in J_{F}(f) \backslash f R$. Then, $a=b g^{\gamma}$ for some $0 \leqslant \gamma<\beta$ and $b \in R$ such that $\operatorname{gcd}(b, g)=1$. Then, $R / g \stackrel{b}{\rightarrow} R / g$ is injective, and so $S / g S \stackrel{b}{\rightarrow} S / g S$ is also injective. Thus, $\operatorname{gcd}(b, g)=1$ in $S$. Hence, $b / g$ is not zero in $S_{g} / S$. Moreover, $b / g=g^{\beta-\gamma-1} a / f \in M$ and it is not zero. Let $g_{1}, \ldots, g_{\ell} \in S$ be irreducible relatively prime elements such that $g=g_{1}^{\beta_{1}} \cdots g_{1}^{\beta_{\ell}}$. We have 
that $b / g_{i}=h b / g \in S_{g_{i}} / S \cap M \backslash\{0\}$, where $h=g_{1}^{\beta_{1}} \cdots g_{i}^{\beta_{i}-1} \cdots g_{1}^{\beta_{\ell}}$. Then, $\mathcal{L}\left(S / g_{i} S, S\right) \subset M$ and so $\mathcal{L}(S / f S, S) \subset M$. Therefore, $J_{F_{S}}(f) \subset J_{F_{R}}(f) S$.

Proposition 4.34. Suppose that $R$ is a local ring. Let $f \in R$. Then $J_{F_{\hat{R}}}(f)=J_{F_{R}}(f) \widehat{R}$, where $\widehat{R}$ denotes the completion of $R$ with respect to the maximal ideal.

Proof. We have that $\mathcal{L}(\widehat{R} / f \widehat{R}, \widehat{R})=\mathcal{L}(R / f R, R) \otimes_{R} \widehat{R}$ [Bli04, Theorem 4.6]. Then,

$$
J_{F_{\hat{R}}}=(\widehat{R} / f \widehat{R}) \cap \mathcal{L}(\widehat{R} / f \widehat{R}, \widehat{R})=((R / f R) \cap \mathcal{L}(R / f R, R)) \otimes_{R} \widehat{R}=J_{F_{R}}(f) \widehat{R} .
$$

Proposition 4.35. Suppose that $(R, \mathfrak{m}, K)$ is local. Let $(S, \eta, L)$ denote a regular $F$-finite ring. Let $R \rightarrow S$ be a flat local morphism such that the closed fiber $S / \mathfrak{m} S$ is regular and $L / K$ is separable. Then, $J_{F_{S}}(f)=J_{F_{R}}(f) S$.

Proof. It suffices to prove that $\mathcal{L}(R / f R, R) \otimes_{R} S=\mathcal{L}(S / f S, S)$. Then, we can assume without loss of generality that $R / f R$ is reduced because the intersection homology depends only on the local cohomology. We have that $J_{F_{\hat{R}}}(f)=J_{F_{R}}(f) \hat{R}$ and $J_{F_{\widehat{S}}}(f)=J_{F_{S}}(f) \widehat{S}$ by Proposition 4.34. In addition, the induced morphism in the completion $\widehat{R} \rightarrow \widehat{S}$ is still a flat local morphism. Since $J_{F_{S}}(f) \subset J_{F_{R}}(f) S$ and $J_{F_{\hat{S}}}(f) \subset J_{F_{\hat{R}}}(f) \widehat{S}$ by Proposition 4.33, $J_{F_{\hat{R}}}(f) \widehat{S} / J_{F_{\widehat{S}}}(f)=\left(J_{F_{R}}(f) S / J_{F_{S}}(f)\right) \otimes_{S} \widehat{S}$. Therefore, we can assume that $R$ and $S$ are complete. In this case, we have that $S / f S$ is reduced and that $\tau(R / f R) S=\tau(S / f S)$ [HH94a, Theorem 7.2]. Therefore, $I_{S}^{j}(f)=I_{R}^{j}(f) S$ and $J_{F_{S}}(f)=J_{F_{R}}(f) S$ by Proposition 4.26 .

We now focus on $F$-Jacobian ideals in polynomial rings over a field. In particular, we study how the ideal is affected by extending the field.

Lemma 4.36. Let $R=K\left[x_{1}, \ldots, x_{n}\right]$, where $K$ is a perfect field. Let $K \rightarrow L$ be an algebraic field extension of $K, S=L\left[x_{1} \ldots, x_{n}\right]$, and $R \rightarrow S$ the map induced by the extension. Suppose that $L$ is an $F$-finite field. Then, $J_{F_{S}}(f)=J_{F_{R}}(f) S$.

Proof. We can assume that $f=g^{\beta}$ where $g$ is an irreducible element in $R$ by 4.16. By Proposition 4.33, It suffices to show that $J_{F_{R}}(R) S \subset J_{F_{S}}(S)$.

There is an inclusion $\phi: R_{f} / R \rightarrow S_{f} / S$, which is induced by $R \rightarrow S$. We take $M=$ $\mathcal{L}(S / f S, S) \cap R_{f} / R$. We claim that $M$ is a $D_{R}$-module of $R_{f} / R$. Since $K$ is perfect,

$$
D_{R}=D(R, \mathbb{Z} / p \mathbb{Z})=\bigcup_{e \in \mathbb{N}} \operatorname{Hom}_{R^{p}}(R, R)=D(R, K)=R\left[\frac{1}{t !} \frac{\partial^{t}}{\partial x_{i}^{t}}\right] .
$$

We note that $D_{R}=D(R, K) \subset D(S, K) \subset D_{S}$, and that $\phi\left(\frac{1}{t !} \frac{\partial^{t}}{\partial x_{i}^{t}} v\right)=\frac{1}{t !} \frac{\partial^{t}}{\partial x_{i}^{t}} \phi(v)$ for every $v \in R_{f} / R$. As a consequence, $\frac{\partial^{t}}{\partial x_{i}^{t}} v \in M$ for every $v \in M$. Therefore, $M$ is a $D_{R}$-module.

Let $I=M \cap R / f R$. We note that

$$
I=\mathcal{L}(S / f S, S) \cap R / f R=\left(J_{F_{S}}(f) / f S\right) \cap R / f R
$$

and that $S / f S$ is an integral extension of $R / f R$ because $L$ is an algebraic extension of $K$. Let $r \in J_{F_{S}}(f) / f S$ not zero, and $a_{j} \in R / f R$ such that $a_{0} \neq 0$ and $r^{n}+a_{n-1} r^{n-1}+\ldots+a_{1} r+a_{0}=0$ in $S / f S$. Then, $r\left(a_{n-1} r^{n-1}+\ldots+a_{1}\right)=-a_{0}$, and so $a_{0} \in I=\left(J_{F_{S}}(f) / f S\right) \cap R / f R$, and 
then $M \neq 0$. Therefore, $\mathcal{L}(R / f R, R) \subset M$ and so $J_{F}(f) / f \subset I$. Let $\pi: R \rightarrow R / f R$ be the quotient morphism. Then,

$$
J_{F_{R}}(f) \subset \pi^{-1}(I)=J_{F_{S}}(f) \cap R, \text { and } J_{F_{R}}(f) S \subset\left(J_{F_{S}}(f) \cap R\right) S \subset J_{F_{S}}(f) .
$$

Lemma 4.37. Let $R=K\left[x_{1}, \ldots, x_{n}\right]$, where $K$ is an $F$-finite field. Let $L=K^{1 / p}, S=$ $L\left[x_{1} \ldots, x_{n}\right]$, and $R \rightarrow S$ the map induced by the extension $K \rightarrow L$. Then $J_{F_{S}}(f)=J_{F_{R}}(f) S$.

Proof. We have that $R \subset S \subset R^{1 / p}$. Then, by Proposition 4.33,

$$
J_{F_{R^{1 / p}}}(f) \subset J_{F_{S}}(f) R^{1 / p} \subset\left(J_{F_{R}}(f) S\right) R^{1 / p}=J_{F_{R}}(f) R^{1 / p} .
$$

Since $J_{F_{R^{1 / p}}}(f)=J_{F_{R}}(f) R^{1 / p}$ by Proposition 4.32,

$$
0=J_{F_{S}}(f) R^{1 / p} /\left(J_{F_{R}}(f) S\right) R^{1 / p}=\left(J_{F_{S}}(f) / J_{F_{R}}(f) S\right) \otimes_{S} R^{1 / p} .
$$

Therefore, $J_{F_{S}}(f)=J_{F_{R}}(f) S$ because $R^{1 / p}$ is a faithfully flat $S$-algebra.

Lemma 4.38. Let $R=K\left[x_{1}, \ldots, x_{n}\right]$, where $K$ is an $F$-finite field. Let $L$ be the perfect closure of $K, S=L\left[x_{1} \ldots, x_{n}\right]$, and $R \rightarrow S$ the map induced by the extension $K \rightarrow L$. Then $J_{F_{S}}(f)=J_{F_{R}}(f) S$.

Proof. We may assume that $f=g^{n}$ for an irreducible $g \in R$ by Proposition 4.16. Let $S^{e}=K^{1 / p^{e}}\left[x_{1}, \ldots, x_{n}\right]$. Let $h_{1}, \ldots, h_{\ell}$ denote a set of generators for $J_{F_{S}}(f)$. In this case, $\left(J_{F_{S}}(f)^{[p]}: f^{p-1}\right)=J_{F_{S}}(f)$. Then there exist $c_{i, j} \in S$ such that $f^{p-1} h_{j}=\sum c_{i, j} h_{j}^{p}$. Since $S=\bigcup_{e} S^{e}$, there exists $m$ such that $c_{i, j}, h_{j} \in S^{m}$. Let $I \subset S^{m}$ be the ideal generated by $h_{1}, \ldots, h_{\ell}$. We note that $I S=J_{F_{S}}(f)$; moreover, $J_{F_{S}}(f) \cap S^{m}=I$ because $S^{e} \rightarrow S$ splits for every $e \in \mathbb{N}$.

We claim that $\left(I^{[p]}: f^{p-1}\right)=I$. We have that $f^{p-1} h_{\ell} \in I^{[p]}$ by our choice of $m$ and so $I \subset\left(I^{[p]}: f^{p-1}\right)$. If $g \in\left(I^{[p]}: f^{p-1}\right)$, then $f^{p-1} g \in I^{[p]} S \subset J_{F_{S}}(f)^{[p]}$ and $g \in J_{F_{S}}(f) \cap S^{m}=I$.

As in the proof of Lemma 4.36, $\left(J_{F_{S}}(f) / f S\right) \cap\left(S^{m} / f S^{m}\right) \neq 0$ and then $J_{F_{S}}(f) \cap S^{m}=$ $I \neq f S$. Therefore, $J_{F_{S}}(f) \subset I$ by Proposition 4.13. Hence,

$$
J_{F_{S}}(f) S \subset I S=J_{F_{S}}(f) \subset J_{F_{S}}(f) S,
$$

and the result follows because

$$
J_{F_{R}}(f) S=\left(J_{F_{R}}(f) S^{m}\right) S=J_{F_{S}}(f) S .
$$

Theorem 4.39. Let $R=K\left[x_{1}, \ldots, x_{n}\right]$, where $K$ is an $F$-finite field. Let $L$ be an algebraic extension of $K$ that is F-finite, $S=L\left[x_{1} \ldots, x_{n}\right]$, and $R \rightarrow S$ the map induced by the extension $K \rightarrow L$. Then $J_{F_{S}}(f)=J_{F_{R}}(f) S$.

Proof. By Proposition 4.33, it suffices to show $J_{F_{R}}(f) S \subset J_{F_{S}}(f)$. Let $K^{*}$ and $L^{*}$ denote the perfect closure of $K$ and $L$ respectively. Let $R^{*}=K^{*}\left[x_{1}, \ldots, x_{n}\right]$ and $S^{*}=L^{*}\left[x_{1}, \ldots, x_{n}\right]$. Then,

$$
\left(J_{F_{R}}(f) S\right) S^{*}=J_{F_{R}}(f) S^{*}=\left(J_{F_{R}}(f) R^{*}\right) S^{*}=J_{F_{R^{*}}}(f) S^{*}=J_{F_{S}}(f)=J_{F_{S}}(f) S^{*}
$$

by Lemma 4.36 and 4.38. Therefore,

$$
\left(J_{F_{R}}(f) S / J_{F_{S}}(f)\right) \otimes_{S} S^{*}=\left(J_{F_{R}}(f) S\right) S^{*} /\left(J_{F_{S}}(f)\right) S^{*}=0 .
$$

Hence $J_{F_{R}}(f) S / J_{F_{S}}(f)=0$ because $S^{*}$ is a faithfully flat $S$-algebra. 
Example 4.40. Let $R=\mathbb{F}_{3}[x, y]$, and $f=x^{2}+y^{2}$ and $\mathfrak{m}=(x, y)$. We have that $\left(\mathfrak{m}^{[p]}\right.$ : $\left.f^{p-1}\right)=\mathfrak{m}$. Then, $J_{F_{R}}(f) \subset \mathfrak{m}$. Let $L=\mathbb{F}_{3}[i]$ the extension of $\mathbb{F}_{3}$ by $\sqrt{-1}, S=L[x, y]$ and $\phi: R \rightarrow S$ be the inclusion given by the field extension. Then, $J_{F_{S}}(f)=(x, y) S$ by Proposition 4.16 because $x^{2}+y^{2}=(x+i y)(x-i y)$. Since $\phi$ is a flat extension, $J_{F_{S}}(f) \subset$ $J_{F_{R}}(f) S$. Then, $\mathfrak{m}=R \cap J_{F_{S}}(f) \subset R \cap J_{F_{R}}(f) S \subset J_{F_{R}}(f)$ Hence, $J_{F}(f)=\mathfrak{m}$.

4.4. Relation with $R\{F\}$-modules and Cartier modules. In this subsection, we discuss two different approaches in which $F$-Jacobian ideals appear. These approaches arise naturally from the relation of test ideals with Cartier modules and $R\{F\}$ modules. We refer to [BB11, Bli13, BS13a, Sch09, ST12] for details about Cartier modules and test ideals, and to [LS01, Sha07, Smi97, Smi95b] for $R\{F\}$-modules and test ideals.

Suppose that $(R, \mathfrak{m}, K)$ is an $F$-finite reduced local Gorenstein ring. Thus $\operatorname{Hom}_{R}\left(R^{1 / p}, R\right)$ has a structure of an $R^{1 / p}$-module given by precomposition of maps: if $\phi \in \operatorname{Hom}_{R}\left(R^{1 / p}, R\right)$, then $r^{1 / p} \cdot \phi$ is defined by the rule $r^{1 / p} \cdot \phi\left(x^{1 / p}\right)=\phi\left(r^{1 / p} x^{1 / p}\right)$. As an $R^{1 / p}$-module, $\operatorname{Hom}_{R}\left(R^{1 / p}, R\right)$ is isomorphic to $R^{1 / p}$ [Fed87, Lemma 1.6], and we pick a generator $\Phi$. We say that an ideal in $I \subset R$ is a Cartier ideal, or an ideal compatible with $\Phi$, if $\Phi\left(I^{1 / p}\right) \subset I$. We point out that this is not the standard definition in the general theory of Cartier modules, and the one given here requires that the ring is Gorenstein.

Suppose that $(R, \mathfrak{m}, K)$ is an $F$-finite regular local ring. Let $f \in \mathfrak{m}$ and $\bar{R}=R / f R$. Let $\Phi \in \operatorname{Hom}_{R}\left(R^{1 / p}, R\right)$ be a generator of this module over $R^{1 / p}$. Let $I, J$ be two ideals of $R$. We point out that $\Phi\left(J^{1 / p}\right) \subset I$ if and only if $J^{1 / p} \subset I R^{1 / p}$, because $R^{1 / p}$ is a free $R$-module. We assume now that $\bar{R}$ is reduced. Then, the map, $\bar{\Phi}_{f}: \bar{R}^{1 / p} \rightarrow \bar{R}$, defined by $\bar{\Phi}\left(x^{1 / p}\right)=\Phi\left(f^{\frac{p-1}{p}} x^{\frac{1}{p}}\right)$ is a generator for $\bar{R}^{1 / p}$ [Fed87, Lemma 1.6 and Corollaries]. We claim that $J_{F}(f) \bar{R}$ is a Cartier ideal of $\bar{R}$. Since $f^{p-1} J_{F}(f) \subset\left(J_{F}(f)\right)^{[p]}$, we deduce that $f^{\frac{p-1}{p}}\left(J_{F}(f) R\right)^{1 / p} \subset J_{F}(f) R^{1 / p}$. Hence, $\bar{\Phi}\left(J_{F}(f) \bar{R}^{1 / p}\right) \subset J_{F}(f) \bar{R}$. Then, $J_{F}(f) \bar{R}$ is a Cartier ideal. By the duality between Cartier modules and $\bar{R}\{F\}$-modules [BB11, Proposition 5.2], we have that $\mathrm{Ann}_{H_{\mathfrak{m}}^{\operatorname{dim}(\bar{R})}(\bar{R})} J_{F}(f) \bar{R}$ is an $\bar{R}\{F\}$-module.

We have that $\bar{\Phi}\left(J^{1 / p}\right) \subset I$ if and only if $J^{1 / p} \subset\left(I \bar{R}^{1 / p}: \bar{R}^{1 / p} f^{\frac{p-1}{p}}\right)$ for any ideals $I, J \subset \bar{R}$. If $I^{\prime}$ is the lift of $I$ to $R$, then $\left(I \bar{R}^{1 / p}: \bar{R}^{1 / p} f^{\frac{p-1}{p}}\right)=\left(I^{\prime} R^{1 / p}: R_{R^{1 / p}} f^{\frac{p-1}{p}}\right) \bar{R}^{1 / p}$ because $f \in I^{\prime}$. Combining this with Proposition 4.26, we obtain $J_{F}(f) \bar{R}$ is the largest Cartier ideal, $I \subset \bar{R}$, such that $\bar{\Phi} \circ \bar{\Phi}^{1 / p} \circ \ldots \circ \bar{\Phi}^{1 / p^{e}}\left(I^{1 / p^{e}}\right) \subset \tau(\bar{R})$ for some $e \in \mathbb{N}$. Furthermore, $\tau(\bar{R})$ and $J_{F}(f) \bar{R}$ are equal up to nilpotency of Cartier modules. By a duality argument, $\operatorname{Ann}_{H_{\mathrm{m}}^{\operatorname{dim}(\bar{R})}(\bar{R})} \tau(\bar{R})$ and $\mathrm{Ann}_{H_{\mathfrak{m}}^{\operatorname{dim}(\bar{R})}(\bar{R})} J_{F}(f) \bar{R}$ are also the same up to nilpotency of $\bar{R}\{F\}$-modules [BB11, Theorem $5.3]$.

4.5. Examples. In this section we present several examples of $F$-Jacobian ideals. These computations are based on previous calculations of the $F$-pure threshold and the classical test ideal.

Proposition 4.41. Let $f \in R$ be an element with an isolated singularity at the maximal ideal $\mathfrak{m}$. If $R_{\mathfrak{m}} / f R_{\mathfrak{m}}$ is F-pure, then

$$
J_{F}(f)= \begin{cases}R & \text { if } R / f R \text { is F-regular } \\ \mathfrak{m} & \text { otherwise }\end{cases}
$$


Proof. Since $R / f R$ has an isolated singularity at $\mathfrak{m}$, we deduce that $J_{F}(f) R_{P}=R_{P}$ for every prime ideal different from $m$. Then, $\mathfrak{m} \subset \sqrt{J_{F}(f)}$.

If $R_{\mathfrak{m}} / f R_{\mathfrak{m}}$ is $F$-regular, then $R / f R$ is $F$-regular, and so $J_{F}(R)=R$ by Corollary 4.29.

If $R_{\mathfrak{m}} / f R_{\mathfrak{m}}$ is not $F$-regular, then $J_{F}(R) \neq R$ by Corollary 4.29. Then, $\mathfrak{m}=\sqrt{J_{F}(f)}$. Since $R_{\mathfrak{m}} / f R_{\mathfrak{m}}$ is $F$-pure, we have that $R_{\mathfrak{m}} / J_{F}(f) R_{\mathfrak{m}}$ is $F$-pure by Lemma 4.30 . Then, $R_{\mathfrak{m}} / J_{F}(f) R_{\mathfrak{m}}$ is a reduced ring. Hence, $J_{F}(f)=\mathfrak{m}$.

Example 4.42. Let $K$ is an $F$-finite field. Let $E$ be an elliptic curve over $K$. We choose a closed immersion of $E$ in $\mathbb{P}_{K}^{2}$ and set $R=K[x, y, z]$, the completed homogeneous coordinate ring of $\mathbb{P}_{K}^{2}$. We take $f \in R$ as the cubic form defining $E$. We know that $f$ has an isolated singularity at $\mathfrak{m}=(x, y, z) R$. If the elliptic curve is ordinary, then $R / f R$ is $F$-pure [Har77, Proposition 4.21] [BS14, Theorem 4.1]. We know that $R / f R$ is never an $F$-regular ring [HH94b, Discussion 7.3b(b), Theorem 7.12]. Then, $J_{F}(f)=\mathfrak{m}$ by Proposition 4.41.

Example 4.43. Let $R=K[x, y, z]$, where is an $F$-finite field of characteristic $p>3$. Let $f=x^{3}+y^{3}+z^{3} \in R$, and $\pi: R \rightarrow R / f R$ be the quotient morphism and $\mathfrak{m}=(x, y, z) R$. We have that $\tau_{f}=\mathfrak{m}$ [Smi95b, Example 6.3]. Then, $\mathfrak{m} \subset J_{F}(f)$ by Proposition 4.26. We know that $R / f R$ is $F$-pure if and only if $p \equiv 1 \bmod 3$. We have that $\left(\mathfrak{m}^{[p]}: f^{p-1}\right)=\mathfrak{m}$ if $p \equiv 1 \bmod 3$, and $\left(\mathfrak{m}^{[p]}: f^{p-1}\right)=R$ if $p \equiv 1 \bmod 2$. Hence, $J_{F}(f)=R$ if $p \equiv 2 \bmod 3$, and $J_{F}(f)=\mathfrak{m}$ if $p \equiv 1 \bmod 3$.

Example 4.44. Let $R=K\left[x_{1}, \ldots, x_{n}\right]$, where $K$ is an $F$-finite field of characteristic $p>0$. Let $f=a_{1} x_{1}^{d_{1}}+\ldots+a_{n} x_{n}^{d_{n}}$, be such that $a_{i} \neq 0$. We have that $R / f R$ has an isolated singularity at the maximal ideal $\mathfrak{m}=\left(x_{1}, \ldots, x_{n}\right)$. If $\frac{1}{d_{1}}+\ldots+\frac{1}{d_{n}}=1$, then $R / f R$ is $F$ pure for $p \gg 0$ [Her15, Theorem 3.1]. In addition, $R$ is not $F$-regular [Gla96, Theorem 3.1] because $f^{p-1}$ is congruent to $c x_{1}^{p^{e}-1} \cdots x_{n}^{p^{e}-1}$ module $\mathfrak{m}^{\left[p^{e}\right]}$ for some element $c \in K \backslash\{0\}$. Hence, $J_{F}(f)=R$ for $p \gg 0$ by Proposition 4.41 .

Remark 4.45. Let $R=K\left[x_{1}, \ldots, x_{n}\right]$ be a polynomial ring and $f \in R$ be such that $R / f R$ is reduced. We can obtain $J_{F}(f)$ from $\tau(R / f R)$ by Proposition 4.26. In the case where $n>3, f=x_{1}^{d}+\ldots x_{n}^{d}$ and $d$ is not divided by the characteristic of $K$, there is an algorithm to compute the test ideal of $R / f R$ [McD03]. Therefore, there is an algorithm to compute $J_{F}(f)$.

Example 4.46. Let $R=K\left[x_{1}, \ldots, x_{n}\right]$, where $K$ is a field of characteristic $p>0$. Let $f=x_{1}^{d}+\ldots+x_{n}^{d}$. This example is based in computations done by McDermott [McD03, Examples 11, 12 and 13].

If $p=2, n=5$ and $d=5, \tau_{f}=\left(x_{i}^{2} x_{j}\right)_{1 \leqslant i, j \leqslant 5}$. Then, $\left(x_{1}^{2}, x_{2}^{2}, x_{3}^{2}, x_{4}^{2}, x_{5}^{2}, x_{1} x_{2} x_{3} x_{4} x_{5}\right) R=$ $\left(\tau_{f}^{[2]}: f\right)$ and $R=\left(\tau_{f}^{[4]}: f^{3}\right)$. Hence, $J_{F}(f)=R$.

If $p=3, n=4$ and $d=7, \tau_{f}=\left(x_{i}^{2} x_{j}^{2}\right)_{1 \leqslant i, j \leqslant 4}$. Then $R=\left(\tau_{f}^{[3]}: f^{2}\right)$ and $J_{F}(f)=R$.

If $p=7, n=5$ and $d=4, \tau_{f}=\left(x_{1}, \ldots, x_{5}\right) R$. Then $R=\left(\tau_{f}^{[7]}: f^{6}\right)$ and $J_{F}(f)=R$.

\section{ACKNOWLEDGMENTS}

We thank Josep Àlvarez Montaner, Angélica Benito, Daniel J. Hernández, Mel Hochster, Mircea Mustaţă, Emily E. Witt, and Wenliang Zhang for insightful mathematical conversations related to this work. We thank Manuel Blickle, Karl Schwede, and Kevin Tucker for 
pointing out missed references. We thank the referee for very helpful comments and suggestions that improved this manuscript, in particular, for pointing out results that lead to a simplified treatment for the F-Jacobian ideals. We also thank Alexis Cook and Rebecca R.G. for their careful reading of this manuscript. The first author thanks the National Council of Science and Technology of Mexico (CONACyT) for its support through grant 210916. The second author was partially supported by the National Science Foundation through grant DMS-1068190.

\section{REFERENCES}

[AF13] Paolo Aluffi and Eleonore Faber. Splayed divisors and their Chern classes. J. Lond. Math. Soc. (2), 88(2):563-579, 2013.

[ÀMBL05] Josep Àlvarez Montaner, Manuel Blickle, and Gennady Lyubeznik. Generators of $D$-modules in positive characteristic. Math. Res. Lett., 12(4):459-473, 2005.

[BB11] Manuel Blickle and Gebhard Böckle. Cartier modules: finiteness results. J. Reine Angew. Math., 661:85-123, 2011.

[BFS13] Angélica Benito, Eleonore Faber, and Karen E. Smith. Measuring singularities with Frobenius: the basics. In Commutative algebra, pages 57-97. Springer, New York, 2013.

[Bli04] Manuel Blickle. The intersection homology D-module in finite characteristic. Math. Ann., 328(3):425-450, 2004.

[Bli08] Manuel Blickle. Minimal $\gamma$-sheaves. Algebra Number Theory, 2(3):347-368, 2008.

[Bli13] Manuel Blickle. Test ideals via algebras of $p^{-e}$-linear maps. J. Algebraic Geom., 22(1):49-83, 2013.

[BM10] Holger Brenner and Paul Monsky. Tight closure does not commute with localization. Ann. of Math. (2), 171(1):571-588, 2010.

[BMS08] Manuel Blickle, Mircea Mustaţă, and Karen E. Smith. Discreteness and rationality of Fthresholds. Michigan Math. J., 57:43-61, 2008. Special volume in honor of Melvin Hochster.

[BMS09] Manuel Blickle, Mircea Mustaţă, and Karen E. Smith. F-thresholds of hypersurfaces. Trans. Amer. Math. Soc., 361(12):6549-6565, 2009.

[BS13a] Manuel Blickle and Karl Schwede. $p^{-1}$-linear maps in algebra and geometry. In Commutative algebra, pages 123-205. Springer, New York, 2013.

[BS13b] M. P. Brodmann and R. Y. Sharp. Local cohomology, volume 136 of Cambridge Studies in Advanced Mathematics. Cambridge University Press, Cambridge, second edition, 2013. An algebraic introduction with geometric applications.

[BS14] Bhargav Bhatt and Anurag K. Singh. The F-pure threshold of a Calabi-Yau hypersurface. Mathematische Annalen, pages 1-17, 2014.

[Fab13] Eleonore Faber. Towards transversality of singular varieties: splayed divisors. Publ. Res. Inst. Math. Sci., 49(3):393-412, 2013.

[Fed87] Richard Fedder. F-purity and rational singularity in graded complete intersection rings. Trans. Amer. Math. Soc., 301(1):47-62, 1987.

[FW89] Richard Fedder and Kei-ichi Watanabe. A characterization of $F$-regularity in terms of $F$-purity. In Commutative algebra (Berkeley, CA, 1987), volume 15 of Math. Sci. Res. Inst. Publ., pages 227-245. Springer, New York, 1989.

[Gla96] Donna Glassbrenner. Strong F-regularity in images of regular rings. Proc. Amer. Math. Soc., 124(2):345-353, 1996.

[Har77] Robin Hartshorne. Algebraic geometry. Springer-Verlag, New York, 1977. Graduate Texts in Mathematics, No. 52.

[Her15] Daniel J. Hernández. F-invariants of diagonal hypersurfaces. Proc. Amer. Math. Soc., 143(1):87$104,2015$.

[HH90] Melvin Hochster and Craig Huneke. Tight closure, invariant theory, and the Briançon-Skoda theorem. J. Amer. Math. Soc., 3(1):31-116, 1990. 
[HH94a] Melvin Hochster and Craig Huneke. F-regularity, test elements, and smooth base change. Trans. Amer. Math. Soc., 346(1):1-62, 1994.

[HH94b] Melvin Hochster and Craig Huneke. Tight closure of parameter ideals and splitting in modulefinite extensions. J. Algebraic Geom., 3(4):599-670, 1994.

[HY03] Nobuo Hara and Ken-Ichi Yoshida. A generalization of tight closure and multiplier ideals. Trans. Amer. Math. Soc., 355(8):3143-3174 (electronic), 2003.

[Kas03] Masaki Kashiwara. D-modules and Microlocal Calculus. American Mathematical Society, 2003.

[LS01] Gennady Lyubeznik and Karen E. Smith. On the commutation of the test ideal with localization and completion. Trans. Amer. Math. Soc., 353(8):3149-3180 (electronic), 2001.

[Lyu97] Gennady Lyubeznik. $F$-modules: applications to local cohomology and $D$-modules in characteristic $p>0$. J. Reine Angew. Math., 491:65-130, 1997.

[McD03] Moira A. McDermott. Test ideals in diagonal hypersurface rings. II. J. Algebra, 264(1):296-304, 2003.

[PS73] C. Peskine and L. Szpiro. Dimension projective finie et cohomologie locale. Applications à la démonstration de conjectures de M. Auslander, H. Bass et A. Grothendieck. Inst. Hautes Études Sci. Publ. Math., (42):47-119, 1973.

[Sch09] Karl Schwede. F-adjunction. Algebra Number Theory, 3(8):907-950, 2009.

[Sha07] Rodney Y. Sharp. Graded annihilators of modules over the Frobenius skew polynomial ring, and tight closure. Trans. Amer. Math. Soc., 359(9):4237-4258 (electronic), 2007.

[Smi94] Karen E. Smith. Tight closure of parameter ideals. Invent. Math., 115(1):41-60, 1994.

[Smi95a] Karen E. Smith. The D-module structure of F-split rings. Math. Res. Lett., 2(4):377-386, 1995.

[Smi95b] Karen E. Smith. Test ideals in local rings. Trans. Amer. Math. Soc., 347(9):3453-3472, 1995.

[Smi97] Karen E. Smith. F-rational rings have rational singularities. Amer. J. Math., 119(1):159-180, 1997.

[ST12] Karl Schwede and Kevin Tucker. A survey of test ideals. In Progress in commutative algebra 2, pages 39-99. Walter de Gruyter, Berlin, 2012.

[Yek92] Amnon Yekutieli. An explicit construction of the Grothendieck residue complex. Astérisque, (208):127, 1992. With an appendix by Pramathanath Sastry.

Department of Mathematics, University of Virginia, Charlottesville, VA 229044137, USA. Email address: 1cn8m@virginia.edu

Department of Mathematics, University of Michigan, Ann Arbor, Mi 48109-1043, USA. Email address: juanfp@umich.edu 